\title{
Sampling and Remote Estimation for the Ornstein-Uhlenbeck Process through Queues: Age of Information and Beyond
}

\author{
Tasmeen Zaman Ornee, Student Member, IEEE and Yin Sun, Senior Member, IEEE
}

\begin{abstract}
Recently, a connection between the age of information and remote estimation error was found in a sampling problem of Wiener processes: If the sampler has no knowledge of the signal being sampled, the optimal sampling strategy is to minimize the age of information; however, by exploiting causal knowledge of the signal values, it is possible to achieve a smaller estimation error. In this paper, we generalize the previous study by investigating a problem of sampling a stationary GaussMarkov process named the Ornstein-Uhlenbeck (OU) process, where we aim to find useful insights for solving the problems of sampling more general signals. The optimal sampling problem is formulated as a constrained continuous-time Markov decision process (MDP) with an uncountable state space. We provide an exact solution to this MDP: The optimal sampling policy is a threshold policy on instantaneous estimation error and the threshold is found. Further, if the sampler has no knowledge of the OU process, the optimal sampling problem reduces to an MDP for minimizing a nonlinear age of information metric. The age-optimal sampling policy is a threshold policy on expected estimation error and the threshold is found. In both problems, the optimal sampling policies can be computed by low-complexity algorithms (e.g., bisection search and Newton's method), and the curse of dimensionality is circumvented. These results hold for (i) general service time distributions of the queueing server and (ii) sampling problems both with and without a sampling rate constraint. Numerical results are provided to compare different sampling policies.
\end{abstract}

Index Terms-Age of information, Ornstein-Uhlenbeck process, sampling policy, threshold policy.

\section{INTRODUCTION}

$\mathbf{T}$ IMELY updates of the system state are of significant importance for state estimation and decision making in networked control and cyber-physical systems, such as UAV navigation, robotics control, mobility tracking, and environment monitoring systems. To evaluate the freshness of state updates, the concept of Age of Information, or simply age, was introduced to measure the timeliness of state samples received from a remote transmitter [1]-[3]. Let $U_{t}$ be the generation time of the freshest received state sample at time $t$. The age of information, as a function of $t$, is defined as $\Delta_{t}=t-U_{t}$, which is the time difference between the freshest samples available at the transmitter and receiver.

Recently, the age of information concept has received significant attention, because of the extensive applications of state updates among systems connected over communication

Tasmeen Zaman Ornee and Yin Sun are with Dept. of ECE, Auburn University, Auburn, AL (emails: tzo0017@auburn.edu, yzs0078@auburn.edu).

This work was supported in part by NSF grant CCF-1813050 and ONR grant N00014-17-1-2417. networks. The states of many systems, such as UAV mobility trajectory and sensor measurements, are in the form of a signal $X_{t}$, that may change slowly at some time and vary more dynamically later. Hence, the time difference described by the age $\Delta_{t}=t-U_{t}$ only partially characterize the variation $X_{t}-X_{U_{t}}$ of the system state, and the state update policy that minimizes the age of information does not minimize the state estimation error. This result was first shown in [4], where a sampling problem of Wiener processes was solved and the optimal sampling policy was shown to have an intuitive structure. As the results therein hold only for signals that can be modeled as a Wiener process, one would wonder how to, and whether it is possible to, extend [4] for handling more general signal models.

In this paper, we generalize [4] by exploring a problem of sampling an Ornstein-Uhlenbeck (OU) process $X_{t}$. From the obtained results, we hope to find useful structural properties of the optimal sampler design that can be potentially applied to more general signal models. The OU process $X_{t}$ is the continuous-time analogue of the well-known first-order autoregressive process, i.e., $\mathrm{AR}(1)$ process. The OU process is defined as the solution to the stochastic differential equation (SDE) [5], [6]

$$
d X_{t}=\theta\left(\mu-X_{t}\right) d t+\sigma d W_{t}
$$

where $\mu, \theta>0$, and $\sigma>0$ are parameters and $W_{t}$ represents a Wiener process. It is the only nontrivial continuous-time process that is stationary, Gaussian, and Markovian [6]. Examples of first-order systems that can be described as the OU process include interest rates, currency exchange rates, and commodity prices (with modifications) [7], control systems such as node mobility in mobile ad-hoc networks, robotic swarms, and UAV systems [8], [9], and physical processes such as the transfer of liquids or gases in and out of a tank [10].

As shown in Fig. 11 samples of an OU process are forwarded to a remote estimator through a channel in a first-come, firstserved (FCFS) fashion. The samples experience i.i.d. random transmission times over the channel, which is caused by random sample size, channel fading, interference, congestions, and etc. For example, UAVs flying close to WiFi access points may suffer from long communication delay and instability issues, because they receive strong interference from the $\mathrm{WiFi}$ access points [11]. We assume that at any time only one sample can be served by the channel. The samples that are waiting to be sent are stored in a queue at the transmitter. Hence, 


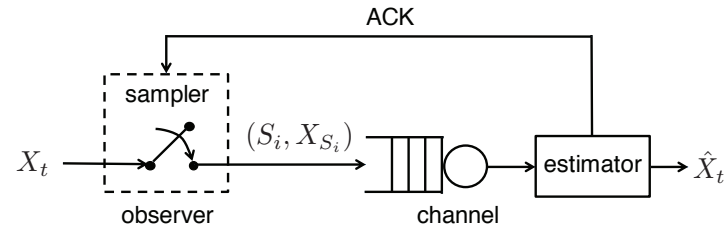

Fig. 1: System model.

the channel is modeled as an FCFS queue with i.i.d. service times. The service time distributions considered in this paper are quite general: they are only required to have a finite mean. This queueing model is helpful to analyze the robustness of remote estimation systems with occasionally long transmission times.

The estimator utilizes causally received samples to construct an estimate $\hat{X}_{t}$ of the real-time signal value $X_{t}$. The quality of remote estimation is measured by the time-average meansquared estimation error, i.e.,

$$
\text { mse }=\limsup _{T \rightarrow \infty} \frac{1}{T} \mathbb{E}\left[\int_{0}^{T}\left(X_{t}-\hat{X}_{t}\right)^{2} d t\right] .
$$

Our goal is to find the optimal sampling policy that minimizes mse by causally choosing the sampling times subject to a maximum sampling rate constraint. In practice, the cost (e.g., energy, CPU cycle, storage) for state updates increases with the average sampling rate. Hence, we are striking to find the optimum tradeoff between estimation error and update cost. In addition, the unconstrained problem is also solved. The contributions of this paper are summarized as follows:

- The optimal sampling problem for minimize the mse under a sampling rate constraint is formulated as a constrained continuous-time Markov decision process (MDP) with an uncountable state space. Because of the curse of dimensionality, such problems are often lack of low-complexity solutions that are arbitrarily accurate. However, we were able to solve this MDP exactly: The optimal sampling policy is proven to be a threshold policy on instantaneous estimation error, where the threshold is a non-linear function $v(\beta)$ of a parameter $\beta$. The value of $\beta$ is equal to the summation of the optimal objective value of the MDP and the optimal Lagrangian dual variable associated to the sampling rate constraint. If there is no sampling rate constraint, the Lagrangian dual variable is zero and hence $\beta$ is exactly the optimal objective value. Among the technical tools developed to prove this result is a free boundary method [12], [13] for finding the optimal stopping time of the OU process.

- The optimal sampler design of Wiener process in [4] is a limiting case of the above result. By comparing the optimal sampling policies of OU process and Wiener process, we find that the threshold function $v(\beta)$ changes according to the signal model, where the parameter $\beta$ is determined in the same way for both signal models.

- Further, we consider a class of signal-agnostic sampling policies, where the sampling times are determined without using knowledge of the signal value of the observed $\mathrm{OU}$ process; the parameters of the $\mathrm{OU}$ process are known. The optimal signal-agnostic sampling problem is equivalent to an MDP for minimizing the time-average of a nonlinear age function $p\left(\Delta_{t}\right)$, which has been solved recently in [14]. The age-optimal sampling policy is a threshold policy on expected estimation error, where the threshold function is simply $v(\beta)=\beta$ and the parameter $\beta$ is determined in the same way as above.

- The above results hold for (i) general service time distributions with a finite mean and (ii) sampling problems both with and without a sampling rate constraint. $\mathrm{Nu}$ merical results suggest that the optimal sampling policy is better than zero-wait sampling and the classic uniform sampling.

One interesting observation from these results is that the threshold function $v(\beta)$ varies with respect to the signal model and sampling problem, but the parameter $\beta$ is determined in the same way.

\section{A. Related Work}

The results in this paper are tightly related to recent studies on the age of information $\Delta_{t}$, e.g., [1], [14]-[35], which does not have a signal model. The average age and average peak age have been analyzed for various queueing systems in, e.g., [1], [18], [20], [21]. The optimality of the Last-Come, First-Served (LCFS) policy, or more generally the Last-Generated, FirstServed (LGFS) policy, was established for various queueing system models in [24]-[26], [30]. Optimal sampling policies for minimizing non-linear age functions were developed in, e.g., [14], [15], [19], [35]. Age-optimal transmission scheduling of wireless networks were investigated in, e.g., [22], [23], [27]-[29], [31], [32].

On the other hand, this paper is also a contribution to the area of remote estimation, e.g., [10], [36]-[41]. In [37], [39], optimal sampling of Wiener processes was studied, where the transmission time from the sampler to the estimator is zero. Optimal sampling of OU processes was also considered in [37], which is solved by discretizing time and using dynamic programming to solve the discrete-time optimal stopping problems. However, our optimal sampler of OU processes is obtained analytically. Remote estimation over several different channel models was recently studied in, e.g., [40], [41]. In [10], [36]-[41], the optimal sampling policies were proven to be threshold policies. Because of the queueing model, our optimal sampling policy has a different structure from those in [10], [36]-[41]. Specifically, in our optimal sampling policy, sampling is suspended when the server is busy and is reactivated once the server becomes idle. In addition, we are able to characterize the threshold precisely. The optimal sampling policy for the Wiener process in [4] is a limiting case of ours. Remote estimation of the Wiener process with random two-way delay was recently considered in [42]. In [43], a jointly optimal sampler, quantizer, and estimator design was found for a class of continuous-time Markov processes under a bit-rate constraint. A recent survey on remote estimation systems was presented in [44]. 


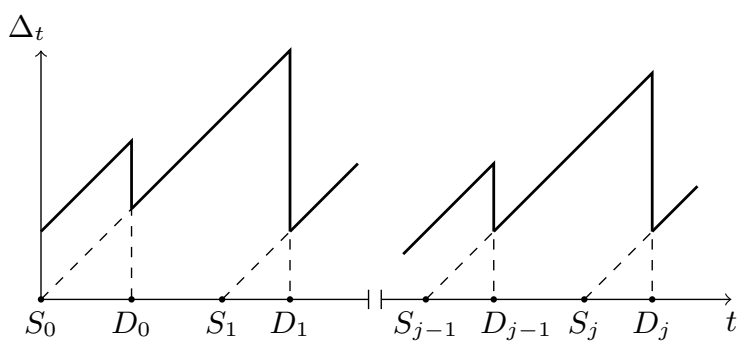

Fig. 2: Evolution of the age $\Delta_{t}$ over time.

\section{MODEL AND FORMULATION}

\section{A. System Model}

We consider the remote estimation system illustrated in Fig. 1, where an observer takes samples from an OU process $X_{t}$ and forwards the samples to an estimator through a communication channel. The channel is modeled as a singleserver FCFS queue with i.i.d. service times. The system starts to operate at time $t=0$. The $i$-th sample is generated at time $S_{i}$ and is delivered to the estimator at time $D_{i}$ with a service time $Y_{i}$, which satisfy $S_{i} \leq S_{i+1}, S_{i}+Y_{i} \leq D_{i}$, $D_{i}+Y_{i+1} \leq D_{i+1}$, and $0<\mathbb{E}\left[Y_{i}\right]<\infty$ for all $i$. Each sample packet $\left(S_{i}, X_{S_{i}}\right)$ contains the sampling time $S_{i}$ and the sample value $X_{S_{i}}$. Let $U_{t}=\max \left\{S_{i}: D_{i} \leq t\right\}$ be the sampling time of the latest received sample at time $t$. The age of information, or simply age, at time $t$ is defined as [1], [2]

$$
\Delta_{t}=t-U_{t}=t-\max \left\{S_{i}: D_{i} \leq t\right\},
$$

which is shown in Fig. 2 Because $D_{i} \leq D_{i+1}, \Delta_{t}$ can be also expressed as

$$
\Delta_{t}=t-S_{i}, \text { if } t \in\left[D_{i}, D_{i+1}\right), i=0,1,2, \ldots
$$

The initial state of the system is assumed to satisfy $S_{0}=0$, $D_{0}=Y_{0}, X_{0}$ and $\Delta_{0}$ are finite constants. The parameters $\mu$, $\theta$, and $\sigma$ in (11) are known at both the sampler and estimator.

Let $I_{t} \in\{0,1\}$ represent the idle/busy state of the server at time $t$. We assume that whenever a sample is delivered, an acknowledgement is sent back to the sampler with zero delay. By this, the idle/busy state $I_{t}$ of the server is known at the sampler. Therefore, the information that is available at the sampler at time $t$ can be expressed as $\left\{X_{s}, I_{s}: 0 \leq s \leq t\right\}$.

\section{B. Sampling Policies}

In causal sampling policies, each sampling time $S_{i}$ is determined based on the up-to-date information that is available at the sampler, without using any future information. In probability theory, such sampling times are represented by stopping times.

To define stopping time precisely, the concepts of $\sigma$-field and filtration are needed. Let us define the $\sigma$-field

$$
\mathcal{N}_{t}=\sigma\left(X_{s}, I_{s}: 0 \leq s \leq t\right),
$$

which is the set of events whose occurrence are determined by the realization of the process $\left\{X_{s}, I_{s}, 0 \leq s \leq t\right\}$ up to time $t$. A filtration is a non-decreasing sequence of $\sigma$-fields. Our analysis requires a strong Markov property, which is satisfied when the filtration is right-continuous. Define

$$
\mathcal{N}_{t}^{+}=\cap_{s>t} \mathcal{N}_{s},
$$

then $\left\{\mathcal{N}_{t}^{+}, t \geq 0\right\}$ is a right-continuous filtration of the information process $\left\{X_{s}, I_{s}, t \geq 0\right\}$ [45]. In a causal sampling policy, each sampling time is a stopping time with respect to $\left\{\mathcal{N}_{t}^{+}, t \geq 0\right\}$, i.e.,

$$
\left\{S_{i} \leq t\right\} \in \mathcal{N}_{t}^{+}, \forall t \geq 0 .
$$

In other words, whether sample $i$ has been generated by time $t$ (i.e., whether $\left\{S_{i} \leq t\right\}$ or $\left\{S_{i}>t\right\}$ ) is determined by the realization of the process $\left\{X_{s}, I_{s}, 0 \leq s \leq t\right\}$ up to time $t$.

Let $\pi=\left(S_{1}, S_{2}, \ldots\right)$ represent a sampling policy. We use $\Pi$ to represent the set of causal sampling policies that satisfy two conditions: (i) Each sampling policy $\pi \in \Pi$ satisfies (6) for all $i$. (ii) The sequence of inter-sampling times $\left\{T_{i}=S_{i+1}-\right.$ $\left.S_{i}, i=0,1, \ldots\right\}$ forms a regenerative process [46, Section 6.1]: There exists an increasing sequence $0 \leq k_{1}<k_{2}<\ldots$ of almost surely finite random integers such that the post $-k_{j}$ process $\left\{T_{k_{j}+i}, i=0,1, \ldots\right\}$ has the same distribution as the post- $k_{0}$ process $\left\{T_{k_{0}+i}, i=0,1, \ldots\right\}$ and is independent of the pre- $k_{j}$ process $\left\{T_{i}, i=0,1, \ldots, k_{j}-1\right\}$; further, we assume that $\mathbb{E}\left[k_{j+1}-k_{j}\right]<\infty, \mathbb{E}\left[S_{k_{1}}\right]<\infty$, and $0<\mathbb{E}\left[S_{k_{j+1}}-\right.$ $\left.S_{k_{j}}\right]<\infty, j=1,2, \ldots[$

From this, we can obtain that $S_{i}$ is finite almost surely for all $i$. We assume that the OU process $\left\{X_{t}, t \geq 0\right\}$ and the service times $\left\{Y_{i}, i=1,2, \ldots\right\}$ are mutually independent, and do not change according to the sampling policy.

A sampling policy $\pi \in \Pi$ is said to be signal-agnostic (signal-aware), if $\pi$ is (not necessarily) independent of $\left\{X_{t}, t \geq 0\right\}$. Let $\Pi_{\text {signal-agnostic }} \subset \Pi$ denote the set of signalagnostic sampling policies, defined as

$$
\Pi_{\text {signal-agnostic }}=\left\{\pi \in \Pi: \pi \text { is independent of }\left\{X_{t}, t \geq 0\right\}\right\} .(7)
$$

\section{MMSE Estimator}

According to (6), $S_{i}$ is a finite stopping time. By using [52, Eq. (3)] and the strong Markov property of the OU process [12, Eq. (4.3.27)], $X_{t}$ is expressed as

$$
\begin{aligned}
X_{t}= & X_{S_{i}} e^{-\theta\left(t-S_{i}\right)}+\mu\left[1-e^{-\theta\left(t-S_{i}\right)}\right] \\
& +\frac{\sigma}{\sqrt{2 \theta}} e^{-\theta\left(t-S_{i}\right)} W_{e^{2 \theta\left(t-S_{i}\right)}-1}, \text { if } t \in\left[S_{i}, \infty\right) .
\end{aligned}
$$

At any time $t \geq 0$, the estimator uses causally received samples to construct an estimate $\hat{X}_{t}$ of the real-time signal value $X_{t}$. The information available to the estimator consists of two parts: (i) $M_{t}=\left\{\left(S_{i}, X_{S_{i}}, D_{i}\right): D_{i} \leq t\right\}$, which contains the sampling time $S_{i}$, sample value $X_{S_{i}}$, and delivery time $D_{i}$ of the samples that have been delivered by time $t$

\footnotetext{
${ }^{1}$ We will optimize $\lim \sup _{T \rightarrow \infty} \mathbb{E}\left[\int_{0}^{T}\left(X_{t}-\hat{X}_{t}\right)^{2} d t\right] / T$, but operationally a nicer criterion is $\limsup _{i \rightarrow \infty} \mathbb{E}\left[\int_{0}^{D_{i}}\left(X_{t}-\hat{X}_{t}\right)^{2} d t\right] / \mathbb{E}\left[D_{i}\right]$. These criteria are corresponding to two definitions of "average cost per unit time" that are widely used in the literature of semi-Markov decision processes. These two criteria are equivalent, if $\left\{T_{1}, T_{2}, \ldots\right\}$ is a regenerative process, or more generally, if $\left\{T_{1}, T_{2}, \ldots\right\}$ has only one ergodic class. If not condition is imposed, however, they are different. The interested readers are referred to [47]-[51] for more discussions.
} 
and (ii) the fact that no sample has been received after the last delivery time $\max \left\{D_{i}: D_{i} \leq t\right\}$. Similar to [4], [37], [53], we assume that the estimator neglects the second part of information 2 Then, as shown in Appendix C, the minimum mean square error (MMSE) estimator is determined by

$$
\begin{aligned}
\hat{X}_{t}=\mathbb{E}\left[X_{t} \mid M_{t}\right]= & X_{S_{i}} e^{-\theta\left(t-S_{i}\right)}+\mu\left[1-e^{-\theta\left(t-S_{i}\right)}\right], \\
& \text { if } t \in\left[D_{i}, D_{i+1}\right), i=0,1,2, \ldots
\end{aligned}
$$

Hence, the estimation error of the MMSE estimator is

$$
\begin{aligned}
X_{t}-\hat{X}_{t}= & \frac{\sigma}{\sqrt{2 \theta}} e^{-\theta t} W_{e^{2 \theta t}-1}, \\
& \text { if } t \in\left[D_{i}, D_{i+1}\right), i=0,1,2, \ldots
\end{aligned}
$$

\section{Problem Formulation}

The goal of this paper is to find the optimal sampling policy that minimizes the mean-squared estimation error subject to an average sampling-rate constraint, which is formulated as the following problem:

$$
\begin{aligned}
\text { mse }_{\mathrm{opt}}=\inf _{\pi \in \Pi} \limsup _{T \rightarrow \infty} \frac{1}{T} \mathbb{E}\left[\int_{0}^{T}\left(X_{t}-\hat{X}_{t}\right)^{2} d t\right] \\
\text { s.t. } \liminf _{n \rightarrow \infty} \frac{1}{n} \mathbb{E}\left[\sum_{i=1}^{n}\left(S_{i+1}-S_{i}\right)\right] \geq \frac{1}{f_{\max }},
\end{aligned}
$$

where mse $_{\text {opt }}$ is the optimum value of (11) and $f_{\max }$ is the maximum allowed sampling rate. When $f_{\max }=\infty$, this problem becomes an unconstrained problem.

\section{MAIN RESUltS}

\section{A. Signal-aware Sampling without Rate Constraint}

Problem (11) is a constrained continuous-time MDP with a continuous state space. However, we found an exact solution to this problem. To present this solution, let us consider an OU process $O_{t}$ with the initial state $O_{t}=0$ and parameter $\mu=0$. According to $[8], O_{t}$ can be expressed as

$$
O_{t}=\frac{\sigma}{\sqrt{2 \theta}} e^{-\theta t} W_{e^{2 \theta t}-1}
$$

Define

$$
\begin{aligned}
& {\text { mse } Y_{i}}=\mathbb{E}\left[O_{Y_{i}}^{2}\right]=\frac{\sigma^{2}}{2 \theta} \mathbb{E}\left[1-e^{-2 \theta Y_{i}}\right], \\
& \text { mse }_{\infty}=\mathbb{E}\left[O_{\infty}^{2}\right]=\frac{\sigma^{2}}{2 \theta} .
\end{aligned}
$$

In the sequel, we will see that $\mathrm{mse}_{Y_{i}}$ and $\mathrm{mse}_{\infty}$ are the lower and upper bounds of $\mathrm{mse}_{\mathrm{opt}}$, respectively. According to 10 and (13)-15, mse $_{Y_{i}}$ represents the estimation error when the

\footnotetext{
${ }^{2}$ We note that this assumption can be removed by considering a joint sampler and estimator design problem. Specifically, it was shown in [10], [36], [38], [40], [41] that when the sampler and estimator are jointly optimized in discrete-time systems, the optimal estimator has the same expression no matter with or without the second part of information. As pointed out in [36 p. 619], such a structure property of the MMSE estimator can be also established for continuous-time systems. The goal of this paper is to find the closed-form expression of the optimal sampler under this assumption. The remaining task of finding the jointly optimal sampler and estimator design can be done by further using the majorization techniques developed in [10], [36], [38], [40], [41]; see [43] for a recent treatment on this task.
}

estimation is made based on a sample that was generated $Y_{i}$ seconds ago, and $\mathrm{mse}_{\infty}$ represents the estimation error for the case that no sample has been delivered to the estimator before. We will also need to use the function

$$
G(x)=\frac{e^{x^{2}}}{x} \int_{0}^{x} e^{-t^{2}} d t=\frac{e^{x^{2}}}{x} \frac{\sqrt{\pi}}{2} \operatorname{erf}(x), x \in[0, \infty),
$$

where $\operatorname{erf}(\cdot)$ is the error function [54], defined as

$$
\operatorname{erf}(x)=\frac{2}{\sqrt{\pi}} \int_{0}^{x} e^{-t^{2}} d t
$$

We first consider the unconstrained optimal sampling problem, i.e., $f_{\max }=\infty$, such that the rate constraint 12 can be removed. In this scenario, the optimal sampler is provided in the following theorem.

Theorem 1. (Sampling without Rate Constraint). If $f_{\max }=$ $\infty$ and the $Y_{i}$ 's are i.i.d. with $0<\mathbb{E}\left[Y_{i}\right]<\infty$, then $\left(S_{1}(\beta), S_{2}(\beta), \ldots\right)$ with a parameter $\beta$ is an optimal solution to (11), where

$$
\begin{gathered}
S_{i+1}(\beta)=\inf \left\{t \geq D_{i}(\beta):\left|X_{t}-\hat{X}_{t}\right| \geq v(\beta)\right\}, \\
D_{i}(\beta)=S_{i}(\beta)+Y_{i}, v(\beta) \text { is defined by } \\
v(\beta)=\frac{\sigma}{\sqrt{\theta}} G^{-1}\left(\frac{\mathrm{mse}_{\infty}-\mathrm{mse}_{Y_{i}}}{\mathrm{mse}_{\infty}-\beta}\right),
\end{gathered}
$$

$G^{-1}(\cdot)$ is the inverse function of $G(\cdot)$ in 16 and $\beta$ is the unique root of

$\mathbb{E}\left[\int_{D_{i}(\beta)}^{D_{i+1}(\beta)}\left(X_{t}-\hat{X}_{t}\right)^{2} d t\right]-\beta \mathbb{E}\left[D_{i+1}(\beta)-D_{i}(\beta)\right]=0$

The optimal objective value to (11) is given by

$$
\text { mse }_{\mathrm{opt}}=\frac{\mathbb{E}\left[\int_{D_{i}(\beta)}^{D_{i+1}(\beta)}\left(X_{t}-\hat{X}_{t}\right)^{2} d t\right]}{\mathbb{E}\left[D_{i+1}(\beta)-D_{i}(\beta)\right]} .
$$

Furthermore, $\beta$ is exactly the optimal value to 111 , i.e., $\beta=$ mse $_{\text {opt }}$.

The proof of Theorem 1 is explained in Section IV] The optimal sampling policy in Theorem 1 has a nice structure. Specifically, the $(i+1)$-th sample is taken at the earliest time $t$ satisfying two conditions: (i) The $i$-th sample has already been delivered by time $t$, i.e., $t \geq D_{i}(\beta)$, and (ii) the estimation error $\left|X_{t}-\hat{X}_{t}\right|$ is no smaller than a pre-determined threshold $v(\beta)$, where $v(\cdot)$ is a non-linear function defined in (19). In Section IV] it is shown that $\mathrm{mse}_{Y_{i}} \leq \beta<\mathrm{mse}_{\infty}$. Further, it is not hard to show that $G(x)$ is strictly increasing on $[0, \infty)$ and $G(0)=1$. Hence, its inverse function $G^{-1}(\cdot)$ and the threshold $v(\beta)$ are properly defined and $v(\beta) \geq 0$.

1) Three Algorithms for Solving (20): We now present three algorithms for computing the root of (20). Because the $S_{i}(\beta)$ 's are stopping times, numerically calculating the expectations in (20) appears to be a difficult task. Nonetheless, this challenge can be solved by resorting to the following lemma, which is obtained by using Dynkin's formula [13, Theorem 7.4.1] and the optional stopping theorem.

\footnotetext{
${ }^{3}$ If $x=0, G(x)$ is defined as its right limit $G(0)=\lim _{x \rightarrow 0^{+}} G(x)=1$.
} 


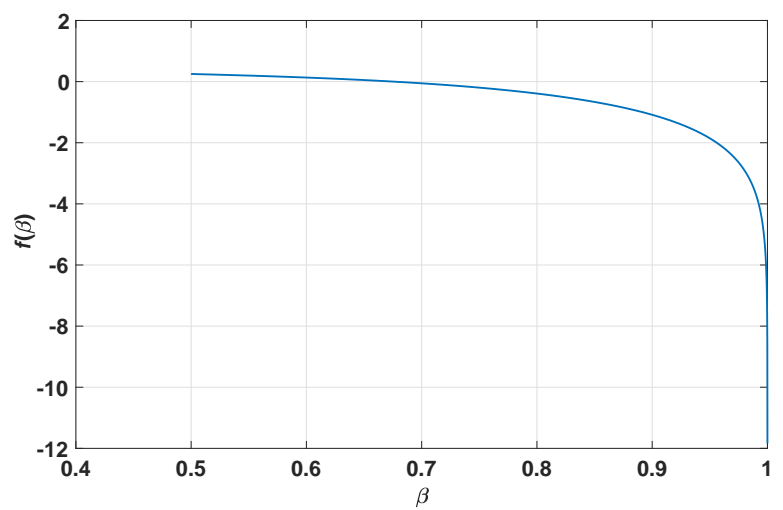

Fig. 3: $f(\beta)$ in 30) for i.i.d. exponential service time with $\mathbb{E}\left[Y_{i}\right]=1$, where the parameters of the OU process are $\sigma=1$ and $\theta=0.5$. For these parameters, $\mathrm{mse}_{Y_{i}}=0.5$ and $\mathrm{mse}_{\infty}=$ 1.

Lemma 1. In Theorem 1 it holds that

$$
\begin{aligned}
& \mathbb{E}\left[D_{i+1}(\beta)-D_{i}(\beta)\right] \\
&= \mathbb{E}\left[\max \left\{R_{1}(v(\beta))-R_{1}\left(O_{Y_{i}}\right), 0\right\}\right]+\mathbb{E}\left[Y_{i}\right], \\
& \mathbb{E}\left[\int_{D_{i}(\beta)}^{D_{i+1}(\beta)}\left(X_{t}-\hat{X}_{t}\right)^{2} d t\right] \\
&=\mathbb{E}\left[\max \left\{R_{2}(v(\beta))-R_{2}\left(O_{Y_{i}}\right), 0\right\}\right] \\
&+\operatorname{mse}_{\infty}\left[\mathbb{E}\left(Y_{i}\right)-\gamma\right]+\mathbb{E}\left[\max \left\{v^{2}(\beta), O_{Y_{i}}^{2}\right\}\right] \gamma,
\end{aligned}
$$

where

$$
\begin{aligned}
& \gamma=\frac{1}{2 \theta} \mathbb{E}\left[1-e^{-2 \theta Y_{i}}\right], \\
& R_{1}(v)=\frac{v^{2}}{\sigma^{2}}{ }_{2} F_{2}\left(1,1 ; \frac{3}{2}, 2 ; \frac{\theta}{\sigma^{2}} v^{2}\right), \\
& R_{2}(v)=-\frac{v^{2}}{2 \theta}+\frac{v^{2}}{2 \theta}{ }_{2} F_{2}\left(1,1 ; \frac{3}{2}, 2 ; \frac{\theta}{\sigma^{2}} v^{2}\right) .
\end{aligned}
$$

Proof. See Appendix []

In (25) and (26), we have used the generalized hypergeometric function, which is defined by [55, Eq. 16.2.1]

$$
\begin{aligned}
& { }_{p} F_{q}\left(a_{1}, a_{2}, \cdots, a_{p} ; b_{1}, b_{2}, \cdots b_{q} ; z\right) \\
= & \sum_{n=0}^{\infty} \frac{\left(a_{1}\right)_{n}\left(a_{2}\right)_{n} \cdots\left(a_{p}\right)_{n}}{\left(b_{1}\right)_{n}\left(b_{2}\right)_{n} \cdots\left(b_{p}\right)_{n}} \frac{z^{n}}{n !},
\end{aligned}
$$

where

$$
\begin{aligned}
& (a)_{0}=1, \\
& (a)_{n}=a(a+1)(a+2) \cdots(a+n-1), n \geq 1 .
\end{aligned}
$$

Using Lemma 1, the expectations in (20) can be evaluated by Monte Carlo simulations of scalar random variables $O_{Y_{i}}$ and $Y_{i}$, which is much simpler than directly simulating the entire random process $\left\{O_{t}, t \geq 0\right\}$.

For notational simplicity, we rewrite (20) as

$$
f(\beta)=f_{1}(\beta)-\beta f_{2}(\beta)=0,
$$

where $f_{1}(\beta)=\mathbb{E}\left[\int_{D_{i}(\beta)}^{D_{i+1}(\beta)}\left(X_{t}-\hat{X}_{t}\right)^{2} d t\right]$ and $f_{2}(\beta)=$
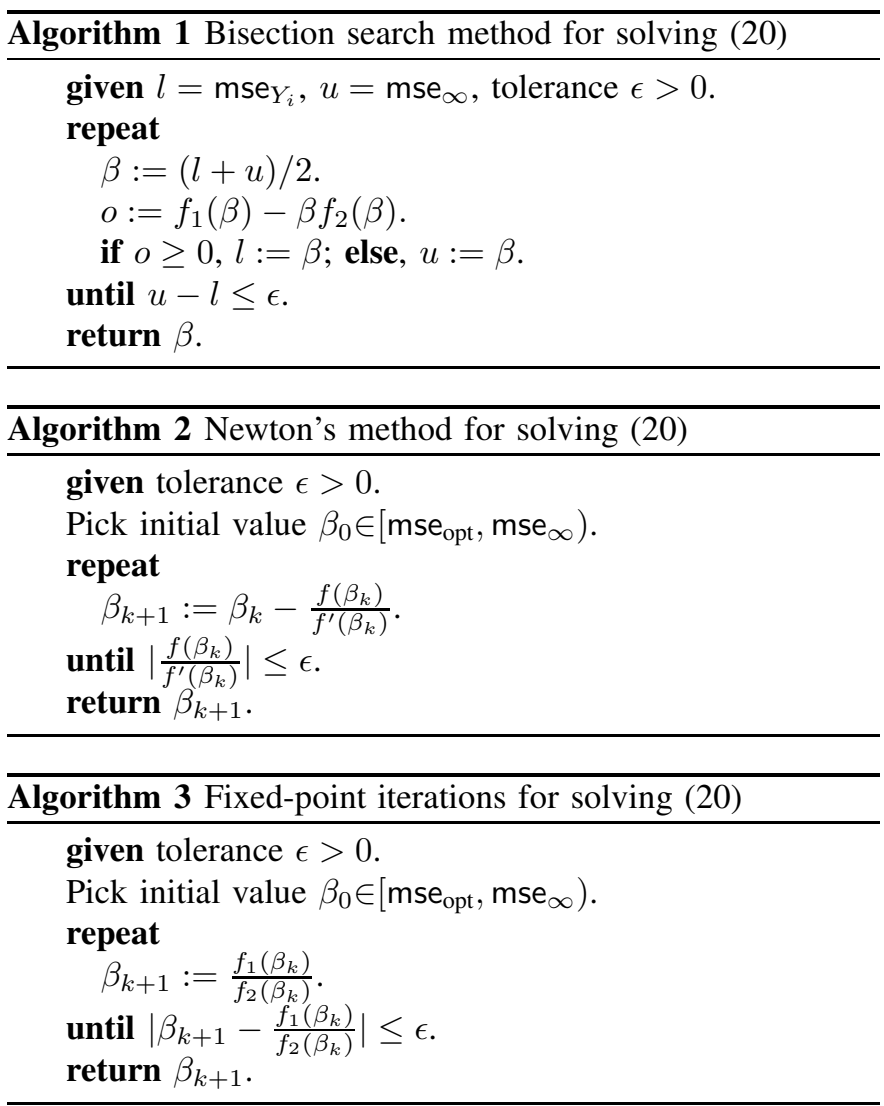

$\mathbb{E}\left[D_{i+1}(\beta)-D_{i}(\beta)\right]$. The function $f(\beta)$ has several nice properties, which are asserted in the following lemma and illustrated in Fig. 3 ,

Lemma 2. The function $f(\beta)$ has the following properties:

(i) $f(\beta)$ is concave, continuous, and strictly decreasing in $\beta$,

(ii) $f\left(\mathrm{mse}_{Y_{i}}\right)>0$ and $\lim _{\beta \rightarrow \mathrm{mse}_{\infty}^{-}} f(\beta)=-\infty$.

\section{Proof. See Appendix A}

The uniqueness of the root of $f(\beta)$ follows immediately from Lemma 2

Because $f(\beta)$ is decreasing and has a unique root, one can use a bisection search method to solve (20), which is illustrated in Algorithm 1 The bisection search method has a globally linear convergence speed.

To achieve an even faster convergence speed, we can use Newton's method [56]

$$
\beta_{k+1}=\beta_{k}-\frac{f\left(\beta_{k}\right)}{f^{\prime}\left(\beta_{k}\right)}
$$

to solve 20), as shown in Algorithm 2 We suggest choosing the initial value $\beta_{0}$ of Newton's method from the set $\left[\mathrm{mse}_{\mathrm{opt}}, \mathrm{mse}_{\infty}\right)$, i.e., $\beta_{0}$ is larger than the root $\mathrm{mse}_{\mathrm{opt}}$. Such an initial value $\beta_{0}$ can be found by taking a few bisection search iterations, or by using the mse of a sub-optimal sampling policy [57]. Because $f(\beta)$ is a concave function, the choice of initial value $\beta_{0} \in\left[\mathrm{mse}_{\mathrm{opt}}, \mathrm{mse}_{\infty}\right)$ ensures that $\beta_{k}$ is a decreasing sequence converging to $\mathrm{mse}_{\mathrm{opt}}$ [58]. Moreover, because $R_{1}(\cdot)$ and $R_{2}(\cdot)$ are twice continuously differentiable, 


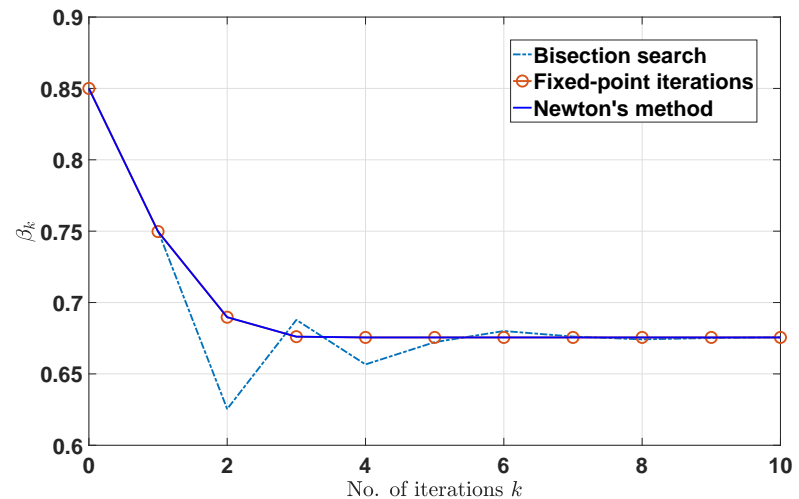

Fig. 4: Convergence of three algorithms for solving (20), where the service times are i.i.d. exponential with mean $\mathbb{E}\left[Y_{i}\right]=1$, the parameters of the OU process are $\sigma=1$ and $\theta=0.5$.

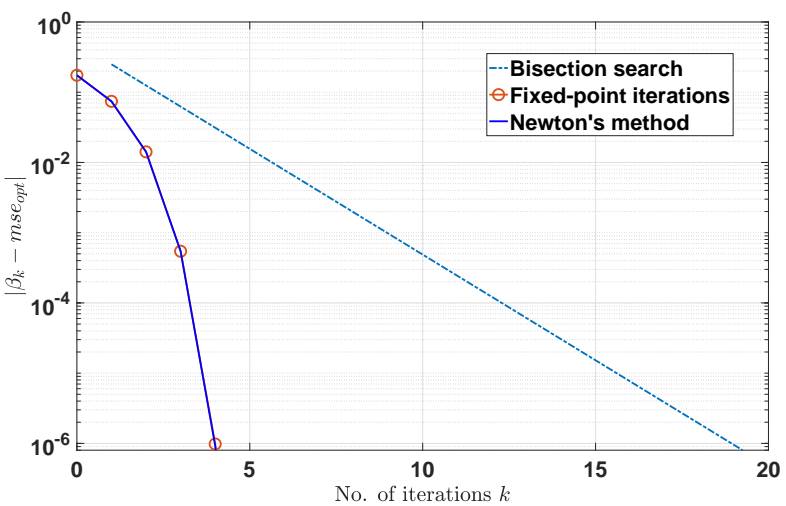

Fig. 5: Convergence of three algorithms for solving 20, where the service times are i.i.d. exponential with mean $\mathbb{E}\left[Y_{i}\right]=1$, the parameters of the OU process are $\sigma=1$ and $\theta=0.5$. For bisection search, we plot the difference $|u-l|$ between the upper bound $u$ and lower bound $l$, which is an upper bound of $\mid \beta_{k}-$ mse $_{\text {opt }} \mid$.

the function $f(\beta)$ is twice continuously differentiable. Therefore, Newton's method is known to have a locally quadratic convergence speed in the neighborhood of the root mse opt $[56$. Chapter 2].

Newton's method requires to compute the gradient $f^{\prime}\left(\beta_{k}\right)$, which can be solved by a finite-difference approximation, as in the secant method [56]. In the sequel, we introduce another approximation approach of Newton's method, which is of independent interest. In Theorem 1, we have shown that

$$
\text { mse }_{\text {opt }}=\underset{\beta \in\left[\operatorname{mse}_{Y_{i}}, \text { mse }_{\infty}\right)}{\operatorname{argmax}} \frac{f_{1}(\beta)}{f_{2}(\beta)} .
$$

Hence, the gradient of $f_{1}(\beta) / f_{2}(\beta)$ is equal to zero at the optimal solution $\beta=$ mse $_{\text {opt }}$, which leads to

$$
f_{1}^{\prime}\left(\mathrm{mse}_{\text {opt }}\right) f_{2}\left(\mathrm{mse}_{\text {opt }}\right)-f_{1}\left(\mathrm{mse}_{\mathrm{opt}}\right) f_{2}^{\prime}\left(\mathrm{mse}_{\mathrm{opt}}\right)=0 .
$$

Therefore,

$$
\mathrm{mse}_{\mathrm{opt}}=\frac{f_{1}\left(\mathrm{mse}_{\mathrm{opt}}\right)}{f_{2}\left(\mathrm{mse}_{\mathrm{opt}}\right)}=\frac{f_{1}^{\prime}\left(\mathrm{mse}_{\mathrm{opt}}\right)}{f_{2}^{\prime}\left(\mathrm{mse}_{\mathrm{opt}}\right)} .
$$

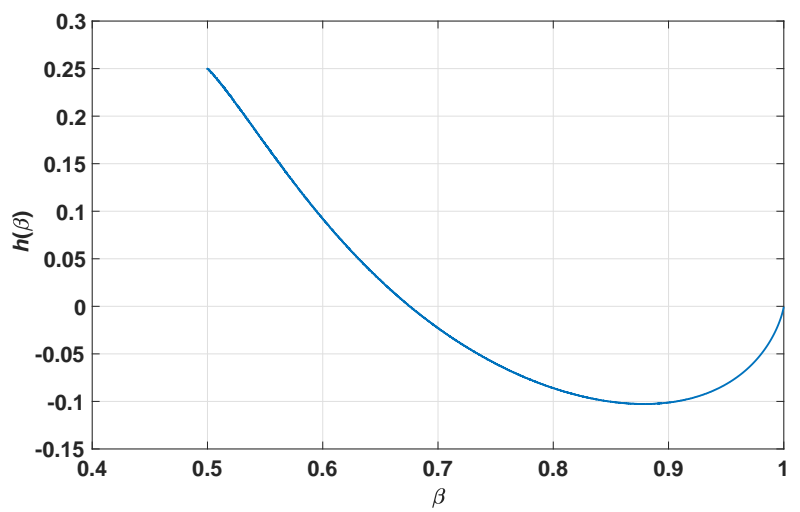

Fig. 6: The function $h(\beta)$ in (36) for i.i.d. exponential service time with $\mathbb{E}\left[Y_{i}\right]=1$, where the parameters of the OU process are $\sigma=1$ and $\theta=0.5$. For these parameters, mse $_{Y_{i}}=0.5$ and $\mathrm{mse}_{\infty}=1$.

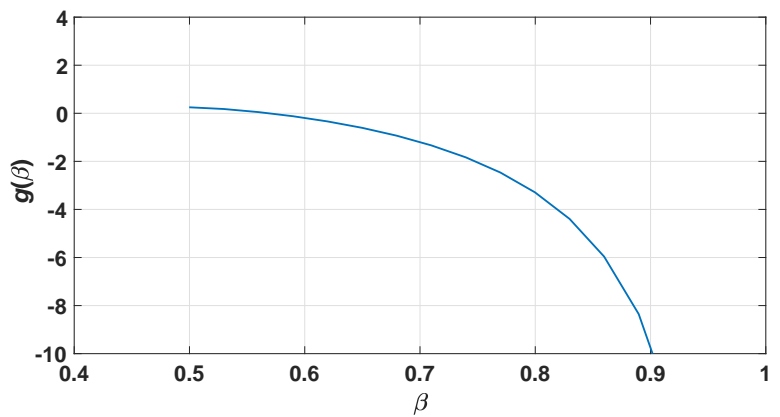

Fig. 7: The function $g(\beta)$ in 40) for i.i.d. exponential service time with $\mathbb{E}\left[Y_{i}\right]=1$ and $f_{\max }=0.8$, where the parameters of the OU process are $\sigma=1$ and $\theta=0.5$. For these parameters, $\mathrm{mse}_{Y_{i}}=0.5$ and $\mathrm{mse}_{\infty}=1$.

Because $f_{1}(\beta)$ and $f_{2}(\beta)$ are smooth functions, when $\beta_{k}$ is in the neighborhood of mse $_{\text {opt }}$, 34 implies that $f_{1}^{\prime}\left(\beta_{k}\right)-$ $\beta_{k} f_{2}^{\prime}\left(\beta_{k}\right) \approx f_{1}^{\prime}\left(\mathrm{mse}_{\text {opt }}\right)-$ mse $_{\text {opt }} f_{2}^{\prime}\left(\mathrm{mse}_{\mathrm{opt}}\right)=0$. Substituting this into 31 , yields

$$
\begin{aligned}
\beta_{k+1} & =\beta_{k}-\frac{f_{1}\left(\beta_{k}\right)-\beta_{k} f_{2}\left(\beta_{k}\right)}{f_{1}^{\prime}\left(\beta_{k}\right)-f_{2}\left(\beta_{k}\right)-\beta_{k} f_{2}^{\prime}\left(\beta_{k}\right)} \\
& \approx \beta_{k}-\frac{f_{1}\left(\beta_{k}\right)-\beta_{k} f_{2}\left(\beta_{k}\right)}{-f_{2}\left(\beta_{k}\right)} \\
& =\frac{f_{1}\left(\beta_{k}\right)}{f_{2}\left(\beta_{k}\right)},
\end{aligned}
$$

which is a fixed-point iterative algorithm (see Algorithm 3) that was recently proposed in [57]. Similar to Newton's method, the fixed-point updates in (35) converge to mse $\mathrm{ept}_{\mathrm{opt}}$ if the initial value $\beta_{0} \in\left[\mathrm{mse}_{\text {opt }}\right.$, mse $_{\infty}$ ). Moreover, (35) has a locally quadratic convergence speed, see [57] for a proof of this result. A numerical comparison of these three algorithms is shown in Fig. 4 and Fig. 5. One can observe that the fixed-point updates and Newton's method converge faster than bisection search.

We note that although (20), and equivalently (30), has a 
unique root $\mathrm{mse}_{\mathrm{opt}}$, the fixed-point equation

$$
h(\beta)=\frac{f_{1}(\beta)}{f_{2}(\beta)}-\beta=\frac{f_{1}(\beta)-\beta f_{2}(\beta)}{f_{2}(\beta)}=0
$$

has two roots $\mathrm{mse}_{\mathrm{opt}}$ and $\mathrm{mse}_{\infty}$. See Fig. 6 for an illustration of the two roots of $h(\beta)$. As shown in Appendix O, the correct root for computing the optimal threshold is $\mathrm{mse}_{\text {opt }}$. Interestingly, Algorithms 11,3 converge to the desired root $\mathrm{mse}_{\mathrm{opt}}$, instead of $\mathrm{mse}_{\infty}$. Finally, we remark that these three algorithms can be used to find the optimal threshold in the age-optimal sampling problem studied in, e.g., [14], [15].

\section{B. Signal-aware Sampling with Rate Constraint}

When the sampling rate constraint (12) is taken into consideration, a solution to (11) is expressed in the following theorem:

Theorem 2. (Sampling with Rate Constraint). If the $Y_{i}$ 's are i.i.d. with $0<\mathbb{E}\left[Y_{i}\right]<\infty$, then (18)-(20) is an optimal solution to (11). The value of $\beta \geq 0$ is determined in two cases: $\beta$ is the unique root of (20) if the root of (20) satisfies

$$
\mathbb{E}\left[D_{i+1}(\beta)-D_{i}(\beta)\right]>1 / f_{\max }
$$

otherwise, $\beta$ is the unique root of

$$
\mathbb{E}\left[D_{i+1}(\beta)-D_{i}(\beta)\right]=1 / f_{\max } .
$$

The optimal objective value to (11) is given by

$$
\mathrm{mse}_{\mathrm{opt}}=\frac{\mathbb{E}\left[\int_{D_{i}(\beta)}^{D_{i+1}(\beta)}\left(X_{t}-\hat{X}_{t}\right)^{2} d t\right]}{\mathbb{E}\left[D_{i+1}(\beta)-D_{i}(\beta)\right]} .
$$

The proof of Theorem 2 is explained in Section IV One can see that Theorem 1 is a special case of Theorem 2 when $f_{\max }=\infty$.

In Theorem 2 the calculation of $\beta$ falls into two cases: In one case, $\beta$ can be computed by solving (20) via Algorithms 1,3. For this case to occur, the sampling rate constraint (12) needs to be inactive at the root of (20). Because $D_{i}(\beta)=$ $S_{i}(\beta)+Y_{i}$, we can obtain $\mathbb{E}\left[D_{i+1}(\beta)-D_{i}(\beta)\right]=\mathbb{E}\left[S_{i+1}(\beta)-\right.$ $\left.S_{i}(\beta)\right]$ and hence (37) holds when the sampling rate constraint (12) is inactive.

In the other case, $\beta$ is selected to satisfy the sampling rate constraint (12) with equality, as required in (38). Before we solve (38), let us first use $f_{2}(\beta)$ to express (38) as

$$
g(\beta)=\frac{1}{f_{\max }}-f_{2}(\beta)=0 .
$$

Lemma 3. The function $g(\beta)$ has the following properties:

(i) $g(\beta)$ is continuous and strictly decreasing in $\beta$,

(ii) $g\left(\operatorname{mse}_{Y_{i}}\right) \geq 0$ and $\lim _{\beta \rightarrow \mathrm{mse}_{\infty}^{-}} g(\beta)=-\infty$ if the root of (20) does not satisfy (37).

Proof. See Appendix B

According to Lemma 3. 38 has a unique root in $\left[\mathrm{mse}_{Y_{i}}, \mathrm{mse}_{\infty}\right)$, which is denoted as $\beta^{*}$. In addition, the numerical results in Fig. 7 suggest that $g(\beta)$ should be concave, for which we do not have a proof.

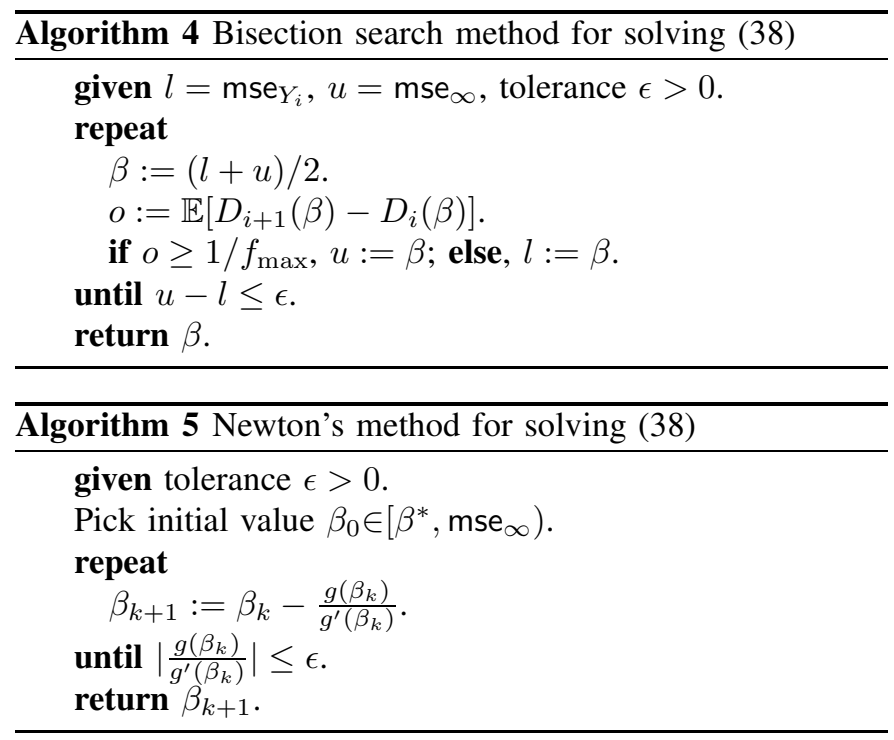

The root $\beta^{*}$ can be solved by using bisection search and Newton's method, which are explained in Algorithms [4,5, respectively. Similar to the discussions in Section III-A1, the convergence of Algorithm 4 is ensured by Lemma 3 . Moreover, if $g(\beta)$ is concave and $\beta_{0} \in\left[\beta^{*}, \mathrm{mse}_{\infty}\right), \beta_{k}$ in Algorithm 5 is a decreasing sequence converging to the root $\beta^{*}$ of (38) [58].

ter

\section{Special Case: Sampling of the Wiener Process}

In the limiting case that $\sigma=1$ and $\theta \rightarrow 0$, the OU process $X_{t}$ in (11) becomes a Wiener process $X_{t}=W_{t}$. In this case, the MMSE estimator in (9) is given by

$$
\hat{X}_{t}=W_{S_{i}}, \text { if } t \in\left[D_{i}, D_{i+1}\right) .
$$

As shown in Appendix E $v(\cdot)$ defined by (19) tends to

$$
v(\beta)=\sqrt{3\left(\beta-\mathbb{E}\left[Y_{i}\right]\right)} .
$$

Theorem 3. If $\sigma=1, \theta \rightarrow 0$, and the $Y_{i}$ 's are i.i.d. with $0<\mathbb{E}\left[Y_{i}\right]<\infty$, then $\left(S_{1}(\beta), S_{2}(\beta), \ldots\right)$ with a parameter $\beta$ is an optimal solution to (11), where

$$
S_{i+1}(\beta)=\inf \left\{t \geq D_{i}(\beta):\left|X_{t}-\hat{X}_{t}\right| \geq \sqrt{3\left(\beta-\mathbb{E}\left[Y_{i}\right]\right)}\right\}
$$

$D_{i}(\beta)=S_{i}(\beta)+Y_{i}$. The value of $\beta \geq 0$ is determined in two cases: $\beta$ is the unique root of

$\mathbb{E}\left[\int_{D_{i}(\beta)}^{D_{i+1}(\beta)}\left(X_{t}-\hat{X}_{t}\right)^{2} d t\right]-\beta \mathbb{E}\left[D_{i+1}(\beta)-D_{i}(\beta)\right]=0$,

if the root of (44) satisfies $\mathbb{E}\left[D_{i+1}(\beta)-D_{i}(\beta)\right]>1 / f_{\max }$; otherwise, $\beta$ is the unique root of $\mathbb{E}\left[D_{i+1}(\beta)-D_{i}(\beta)\right]=$ $1 / f_{\max }$. The optimal objective value to (11) is given by

$$
\mathrm{mse}_{\mathrm{opt}}=\frac{\mathbb{E}\left[\int_{D_{i}(\beta)}^{D_{i+1}(\beta)}\left(X_{t}-\hat{X}_{t}\right)^{2} d t\right]}{\mathbb{E}\left[D_{i+1}(\beta)-D_{i}(\beta)\right]} .
$$

Theorem 3 is an alternative form of Theorem 1 in [4] and hence its proof is omitted. The benefit of the new expression 
in Theorem 3 is that it allows to character $\beta$ based on the

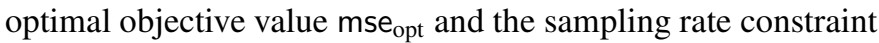
(12), in the same way as in Theorems 1.2. This appears to be more fundamental than the expression in [4]. The new form of optimal sampling policy of Wiener processes was also discovered in [42] without considering the constraint on (12).

\section{Signal-agnostic Sampling}

In signal-agnostic sampling policies, the sampling times $S_{i}$ are determined based only on the service times $Y_{i}$, but not on the observed OU process $\left\{X_{t}, t \geq 0\right\}$.

Lemma 4. If $\pi \in \Pi_{\text {signal-agnostic, }}$ then the mean-squared estimation error of the $O U$ process $X_{t}$ at time $t$ is

$$
p\left(\Delta_{t}\right)=\mathbb{E}\left[\left(X_{t}-\hat{X}_{t}\right)^{2} \mid \pi, Y_{1}, Y_{2}, \ldots\right]=\frac{\sigma^{2}}{2 \theta}\left(1-e^{-2 \theta \Delta_{t}}\right),
$$

which is a strictly increasing function of the age $\Delta_{t}$.

Proof. See Appendix D,

According to Lemma 4 for every policy $\pi \in \Pi_{\text {signal-agnostic }}$,

$$
\mathbb{E}\left[\int_{0}^{T}\left(X_{t}-\hat{X}_{t}\right)^{2} d t\right]=\mathbb{E}\left[\int_{0}^{T} p\left(\Delta_{t}\right) d t\right] .
$$

Hence, minimizing the mean-squared estimation error among signal-agnostic sampling policies can be formulated as the following MDP for minimizing the expected time-average of the nonlinear age function $p\left(\Delta_{t}\right)$ in (46):

$$
\begin{array}{r}
\text { mse }_{\text {age-opt }}=\inf _{\pi \in \Pi_{\text {signal-agnosic }}} \limsup _{T \rightarrow \infty} \frac{1}{T} \mathbb{E}\left[\int_{0}^{T} p\left(\Delta_{t}\right) d t\right] \\
\text { s.t. } \quad \liminf _{n \rightarrow \infty} \frac{1}{n} \mathbb{E}\left[\sum_{i=1}^{n}\left(S_{i+1}-S_{i}\right)\right] \geq \frac{1}{f_{\max }},
\end{array}
$$

where $\mathrm{mse}_{\text {age-opt }}$ is the optimal value of (48). By (46), $p\left(\Delta_{t}\right)$ and mse $_{\text {age-opt }}$ are bounded. Because $\Pi_{\text {signal-agnostic }} \subset \Pi$, it follows immediately that $\mathrm{mse}_{\text {opt }} \leq \mathrm{mse}_{\text {age-opt }}$.

Problem (48) is one instance of the problems recently solved in Corollary 3 of [14] for general strictly increasing functions $p(\cdot)$. From this, a solution to (48) for signal-agnostic sampling is given by

Theorem 4. If the $Y_{i}$ 's are i.i.d. with $0<\mathbb{E}\left[Y_{i}\right]<\infty$, then $\left(S_{1}(\beta), S_{2}(\beta), \ldots\right)$ with a parameter $\beta$ is an optimal solution to (48), where

$$
S_{i+1}(\beta)=\inf \left\{t \geq D_{i}(\beta): \mathbb{E}\left[\left(X_{t+Y_{i+1}}-\hat{X}_{t+Y_{i+1}}\right)^{2}\right] \geq \beta\right\},
$$

$D_{i}(\beta)=S_{i}(\beta)+Y_{i}$ and $\beta$ is the unique root of

$\mathbb{E}\left[\int_{D_{i}(\beta)}^{D_{i+1}(\beta)}\left(X_{t}-\hat{X}_{t}\right)^{2} d t\right]-\beta \mathbb{E}\left[D_{i+1}(\beta)-D_{i}(\beta)\right]=0,(51)$

if the root of 511 satisfies $\mathbb{E}\left[D_{i+1}(\beta)-D_{i}(\beta)\right]>1 / f_{\max }$; otherwise, $\beta$ is the unique root of

$$
\mathbb{E}\left[D_{i+1}(\beta)-D_{i}(\beta)\right]=1 / f_{\max } .
$$

The optimal objective value to (48) is given by

$$
\text { mse }_{\text {age-opt }}=\frac{\mathbb{E}\left[\int_{D_{i}(\beta)}^{D_{i+1}(\beta)}\left(X_{t}-\hat{X}_{t}\right)^{2} d t\right]}{\mathbb{E}\left[D_{i+1}(\beta)-D_{i}(\beta)\right]} .
$$

Theorem 4 follows from Corollary 3 of [14] and Lemma 4 Similar to the case of signal-aware sampling, the roots of (51) and (52) can be solved by using Algorithms 10,5 In fact, Algorithms 15 can be used for minimizing general nondecreasing age penalty [14].

\section{E. Discussions of the Results}

The difference among Theorems 14 is only in the expressions (18), (43), (50) of threshold policies. In signalaware sampling policies (18) and (43), the sampling time is determined by the instantaneous estimation error $\left|X_{t}-\hat{X}_{t}\right|$, and the threshold function $v(\cdot)$ is determined by the specific signal model. In the signal-agnostic sampling policy (50), the sampling time is determined by the expected estimation error $\mathbb{E}\left[\left(X_{t+Y_{i+1}}-\hat{X}_{t+Y_{i+1}}\right)^{2}\right]$ at time $t+Y_{i+1}$. We note that if $t=S_{i+1}(\beta)$, then $t+Y_{i+1}=S_{i+1}(\beta)+Y_{i+1}=D_{i+1}(\beta)$ is the delivery time of the new sample. Hence, (50) requires that the expected estimation error upon the delivery of the new sample is no less than $\beta$. The parameter $\beta$ in Theorems 1.4 is determined by the optimal objective value and the sampling rate constraint in the same manner. Later on in (69), we will further see that $\beta$ is exactly equal to the summation of the optimal objective value of the MDP and the optimal Lagrangian dual variable associated to the sampling rate constraint. Finally, it is worth noting that Theorems 1 . 4 hold for all distributions of the service times $Y_{i}$ satisfying $0<\mathbb{E}\left[Y_{i}\right]<\infty$, and for both constrained and unconstrained sampling problems.

\section{Proof of the Main Results}

We first provide the proof of Theorem 2 After that Theorem 1 follows immediately because it is a special case of Theorem 2. We prove Theorem 2 in four steps: (i) We first show that sampling should be suspended when the server is busy, which can be used to simplify (11). (ii) We use an extended Dinkelbach's method [59] and Lagrangian duality method to decompose the simplified problem into a series of mutually independent per-sample MDP. (iii) We utilize the free boundary method from optimal stopping theory [12] to solve the per-sample MDPs analytically. (iv) Finally, we use a geometric multiplier method [60] to show that the duality gap is zero. The above proof framework is an extension to that used in [4], [14], and the most challenging part is Step (iii).

\section{A. Preliminaries}

The OU process $O_{t}$ in (13) with initial state $O_{t}=0$ and parameter $\mu=0$ is the solution to the SDE

$$
d O_{t}=-\theta O_{t} d t+\sigma d W_{t} .
$$

In addition, the infinitesimal generator of $O_{t}$ is [61, Eq. A1.22]

$$
\mathcal{G}=-\theta u \frac{\partial}{\partial u}+\frac{\sigma^{2}}{2} \frac{\partial^{2}}{\partial u^{2}} \text {. }
$$


According to (8) and (9), the estimation error $\left(X_{t}-\hat{X}_{t}\right)$ is of the same distribution with $O_{t-S_{i}}$, if $t \in\left[D_{i}, D_{i+1}\right)$. By using Dynkin's formula and the optional stopping theorem, we obtain the following lemma.

Lemma 5. Let $\tau \geq 0$ be a stopping time of the OU process $O_{t}$ with $\mathbb{E}[\tau]<\infty$, then

$$
\mathbb{E}\left[\int_{0}^{\tau} O_{t}^{2} d t\right]=\mathbb{E}\left[\frac{\sigma^{2}}{2 \theta} \tau-\frac{1}{2 \theta} O_{\tau}^{2}\right] .
$$

If, in addition, $\tau$ is the first exit time of a bounded set, then

$$
\begin{aligned}
& \mathbb{E}[\tau]=\mathbb{E}\left[R_{1}\left(O_{\tau}\right)\right], \\
& \mathbb{E}\left[\int_{0}^{\tau} O_{t}^{2} d t\right]=\mathbb{E}\left[R_{2}\left(O_{\tau}\right)\right],
\end{aligned}
$$

where $R_{1}(\cdot)$ and $R_{2}(\cdot)$ are defined in (25) and (26), respectively.

\section{Proof. See Appendix F}

\section{B. Suspend Sampling When the Server is Busy}

By using the strong Markov property of the OU process $X_{t}$ and the orthogonality principle of MMSE estimation, we obtain the following useful lemma:

Lemma 6. Suppose that a feasible sampling policy for problem (11) is $\pi$, in which at least one sample is taken when the server is busy processing an earlier generated sample. Then, there exists another feasible policy $\pi^{\prime}$ for problem (11) which has a smaller estimation error than policy $\pi$. Therefore, in (11), it is suboptimal to take a new sample before the previous sample is delivered.

Proof. See Appendix G

A similar result was obtained in [4] for the sampling of Wiener processes. By Lemma 6 there is no loss to consider a sub-class of sampling policies $\Pi_{1} \subset \Pi$ such that each sample is generated and sent out after all previous samples are delivered, i.e.,

$$
\Pi_{1}=\left\{\pi \in \Pi: S_{i}=G_{i} \geq D_{i-1} \text { for all } i\right\} .
$$

For any policy $\pi \in \Pi_{1}$, the information used for determining $S_{i}$ includes: (i) the history of signal values $\left(X_{t}: t \in\left[0, S_{i}\right]\right)$ and (ii) the service times $\left(Y_{1}, \ldots, Y_{i-1}\right)$ of previous samples. Let us define the $\sigma$-fields $\mathcal{F}_{t}=\sigma\left(X_{s}: s \in[0, t]\right)$ and $\mathcal{F}_{t}^{+}=\cap_{r>t} \mathcal{F}_{r}$. Then, $\left\{\mathcal{F}_{t}^{+}, t \geq 0\right\}$ is the filtration (i.e., a non-decreasing and right-continuous family of $\sigma$-fields) of the OU process $X_{t}$. Given the service times $\left(Y_{1}, \ldots, Y_{i-1}\right)$ of previous samples, $S_{i}$ is a stopping time with respect to the filtration $\left\{\mathcal{F}_{t}^{+}, t \geq 0\right\}$ of the OU process $X_{t}$, that is

$$
\left[\left\{S_{i} \leq t\right\} \mid Y_{1}, \ldots, Y_{i-1}\right] \in \mathcal{F}_{t}^{+} .
$$

Hence, the policy space $\Pi_{1}$ can be expressed as

$$
\begin{array}{r}
\Pi_{1}=\left\{S_{i}:\left[\left\{S_{i} \leq t\right\} \mid Y_{1}, \ldots, Y_{i-1}\right] \in \mathcal{F}_{t}^{+},\right. \\
\left.T_{i} \text { is a regenerative process }\right\} .
\end{array}
$$

Let $Z_{i}=S_{i+1}-D_{i} \geq 0$ represent the waiting time between the delivery time $D_{i}$ of the $i$-th sample and the generation time
$S_{i+1}$ of the $(i+1)$-th sample. Then, $S_{i}=\sum_{j=0}^{i-1}\left(Y_{j}+Z_{j}\right)$ and $D_{i}=\sum_{j=0}^{i-1}\left(Y_{j}+Z_{j}\right)+Y_{i}$ for each $i=1,2, \ldots$ Given $\left(Y_{0}, Y_{1}, \ldots\right),\left(S_{1}, S_{2}, \ldots\right)$ is uniquely determined by $\left(Z_{0}, Z_{1}, \ldots\right)$. Hence, one can also use $\pi=\left(Z_{0}, Z_{1}, \ldots\right)$ to represent a sampling policy.

Because $\left\{X_{t}-\hat{X}_{t}, t \in\left[D_{i}, D_{i+1}\right)\right\}$ and $\left\{O_{t-S_{i}}, t \in\right.$ $\left.\left[D_{i}, D_{i+1}\right)\right\}$ are of the same distribution, for each $i=1,2, \ldots$,

$$
\begin{aligned}
& \mathbb{E}\left[\int_{D_{i}}^{D_{i+1}}\left(X_{t}-\hat{X}_{t}\right)^{2} d t\right] \\
= & \mathbb{E}\left[\int_{D_{i}}^{D_{i+1}} O_{t-S_{i}}^{2} d t\right]=\mathbb{E}\left[\int_{Y_{i}}^{Y_{i}+Z_{i}+Y_{i+1}} O_{s}^{2} d s\right] .
\end{aligned}
$$

Because $T_{i}$ is a regenerative process, the renewal theory [62] tells us that $\frac{1}{n} \mathbb{E}\left[S_{n}\right]$ is a convergent sequence and

$$
\begin{aligned}
& \limsup _{T \rightarrow \infty} \frac{1}{T} \mathbb{E}\left[\int_{0}^{T}\left(X_{t}-\hat{X}_{t}\right)^{2} d t\right] \\
= & \lim _{n \rightarrow \infty} \frac{\mathbb{E}\left[\int_{0}^{D_{n}}\left(X_{t}-\hat{X}_{t}\right)^{2} d t\right]}{\mathbb{E}\left[D_{n}\right]} \\
= & \lim _{n \rightarrow \infty} \frac{\sum_{i=1}^{n} \mathbb{E}\left[\int_{Y_{i}}^{Y_{i}+Z_{i}+Y_{i+1}} O_{s}^{2} d s\right]}{\sum_{i=1}^{n} \mathbb{E}\left[Y_{i}+Z_{i}\right]} .
\end{aligned}
$$

Hence, (11) can be rewritten as the following MDP:

$$
\begin{aligned}
\mathrm{mse}_{\mathrm{opt}}= & \inf _{\pi \in \Pi_{1}} \lim _{n \rightarrow \infty} \frac{\sum_{i=1}^{n} \mathbb{E}\left[\int_{Y_{i}}^{Y_{i}+Z_{i}+Y_{i+1}} O_{s}^{2} d s\right]}{\sum_{i=1}^{n} \mathbb{E}\left[Y_{i}+Z_{i}\right]} \\
& \text { s.t. } \lim _{n \rightarrow \infty} \frac{1}{n} \sum_{i=1}^{n} \mathbb{E}\left[Y_{i}+Z_{i}\right] \geq \frac{1}{f_{\max }},
\end{aligned}
$$

where $\mathrm{mse}_{\mathrm{opt}}$ is the optimal value of (63).

\section{Reformulation of Problem (63)}

In order to solve (63), let us consider the following MDP with a parameter $c \geq 0$ :

$$
\begin{aligned}
h(c)= & \inf _{\pi \in \Pi_{1}} \lim _{n \rightarrow \infty} \frac{1}{n} \sum_{i=1}^{n} \mathbb{E}\left[\int_{Y_{i}}^{Y_{i}+Z_{i}+Y_{i+1}} O_{s}^{2} d s-c\left(Y_{i}+Z_{i}\right)\right] \\
& \text { s.t. } \lim _{n \rightarrow \infty} \frac{1}{n} \sum_{i=1}^{n} \mathbb{E}\left[Y_{i}+Z_{i}\right] \geq \frac{1}{f_{\max }},
\end{aligned}
$$

where $h(c)$ is the optimum value of (64). Similar with Dinkelbach's method [59] for nonlinear fractional programming, the following lemma holds for the MDP (63):

Lemma 7. [4] The following assertions are true:

(a). $\mathrm{mse}_{\mathrm{opt}} \gtreqless c$ if and only if $h(c) \gtreqless 0$.

(b). If $h(c)=0$, the solutions to (63) and (64) are identical.

Hence, the solution to (63) can be obtained by solving (64) and seeking $c=$ mse $_{\mathrm{opt}} \geq 0$ such that

$$
h\left(\mathrm{mse}_{\mathrm{opt}}\right)=0 .
$$




\section{Lagrangian Dual Problem of 64}

Next, we use the Lagrangian dual approach to solve 64 with $c=$ mse $_{\text {opt }}$. We define the Lagrangian associated with (64) as

$$
\begin{aligned}
& L(\pi ; \lambda) \\
= & \lim _{n \rightarrow \infty} \frac{1}{n} \sum_{i=1}^{n} \mathbb{E}\left[\int_{Y_{i}}^{Y_{i}+Z_{i}+Y_{i+1}} O_{s}^{2} d s-\left(\mathrm{mse}_{\mathrm{opt}}+\lambda\right)\left(Y_{i}+Z_{i}\right)\right] \\
& +\frac{\lambda}{f_{\max }}
\end{aligned}
$$

where $\lambda \geq 0$ is the dual variable. Let

$$
e(\lambda)=\inf _{\pi \in \Pi_{1}} L(\pi ; \lambda) .
$$

Then, the dual problem of 64 is defined by

$$
d=\max _{\lambda \geq 0} e(\lambda)
$$

where $d$ is the optimum value of 68. Weak duality [60] implies $d \leq h\left(\right.$ mse $\left._{\text {opt }}\right)$. In Section IV-F, we will establish strong duality, i.e., $d=h\left(\mathrm{mse}_{\mathrm{opt}}\right)$.

In the sequel, we decompose 675 into a sequence of mutually independent per-sample MDPs. Let us define

$$
\beta=\mathrm{mse}_{\mathrm{opt}}+\lambda \text {. }
$$

As shown in Appendix $[$ by using Lemma 5, we can obtain

$$
\begin{aligned}
& \mathbb{E}\left[\int_{Y_{i}}^{Y_{i}+Z_{i}+Y_{i+1}} O_{s}^{2} d s-\beta\left(Y_{i}+Z_{i}\right)\right] \\
= & \mathbb{E}\left[\int_{Y_{i}}^{Y_{i}+Z_{i}}\left(O_{s}^{2}-\beta\right) d s+\gamma O_{Y_{i}+Z_{i}}^{2}\right] \\
+ & \frac{\sigma^{2}}{2 \theta}\left[\mathbb{E}\left(Y_{i+1}\right)-\gamma\right]-\beta \mathbb{E}\left[Y_{i+1}\right],
\end{aligned}
$$

where $\gamma$ is defined in (24). For any $s \geq 0$, define the $\sigma$ fields $\mathcal{F}_{t}^{s}=\sigma\left(O_{s+r}-O_{s}: r \in[0, t]\right)$ and the rightcontinuous filtration $\mathcal{F}_{t}^{s+}=\cap_{r>t} \mathcal{F}_{r}^{s}$. Then, $\left\{\mathcal{F}_{t}^{s+}, t \geq 0\right\}$ is the filtration of the time-shifted OU process $\left\{O_{s+t}-O_{s}, t \in\right.$ $[0, \infty)\}$. Define $\mathfrak{M}_{s}$ as the set of integrable stopping times of $\left\{O_{s+t}-O_{s}, t \in[0, \infty)\right\}$, i.e.,

$$
\mathfrak{M}_{s}=\left\{\tau \geq 0:\{\tau \leq t\} \in \mathcal{F}_{t}^{s+}, \mathbb{E}[\tau]<\infty\right\} .
$$

By using a sufficient statistic of (67), we can obtain

Lemma 8. An optimal solution $\left(Z_{0}, Z_{1}, \ldots\right)$ to (67) satisfies

$$
\inf _{Z_{i} \in \mathfrak{M}_{Y_{i}}} \mathbb{E}\left[\int_{Y_{i}}^{Y_{i}+Z_{i}}\left(O_{s}^{2}-\beta\right) d s+\gamma O_{Y_{i}+Z_{i}}^{2} \mid O_{Y_{i}}, Y_{i}\right],
$$

where $\beta \geq 0$ and $\gamma \geq 0$ are defined in (69) and (24), respectively.

Proof. See Appendix Ј. 72).

\section{E. Analytical Solution to Per-Sample MDP (72)}

We solve (72) by using the free-boundary approach for optimal stopping problems [12].

Let us consider an OU process $V_{t}$ with initial state $V_{0}=v$ and parameter $\mu=0$. Define the $\sigma$-fields $\mathcal{F}_{t}^{V}=\sigma\left(V_{s}: s \in\right.$ $[0, t]), \mathcal{F}_{t}^{V+}=\cap_{r>t} \mathcal{F}_{r}^{V}$, and the filtration $\left\{\mathcal{F}_{t}^{V+}, t \geq 0\right\}$ associated to $\left\{V_{t}, t \geq 0\right\}$. Define $\mathfrak{M}_{V}$ as the set of integrable stopping times of $\left\{V_{t}, t \in[0, \infty)\right\}$, i.e.,

$$
\mathfrak{M}_{V}=\left\{\tau \geq 0:\{\tau \leq t\} \in \mathcal{F}_{t}^{V+}, \mathbb{E}[\tau]<\infty\right\} .
$$

Our goal is to solve the following optimal stopping problem for any given initial state $v \in \mathbb{R}$

$$
\sup _{\tau \in \mathfrak{M}_{V}} \mathbb{E}_{v}\left[-\gamma V_{\tau}^{2}-\int_{0}^{\tau}\left(V_{s}^{2}-\beta\right) d s\right],
$$

where $\mathbb{E}_{v}[\cdot]$ is the conditional expectation for given initial state $V_{0}=v, \gamma$ and $\beta$ are given by (24) and 69), respectively. Hence, (72) is one instance of (74) with $v=O_{Y_{i}}$, where the supremum is taken over all stopping times $\tau$ of $V_{t}$. In this subsection, we focus on the case that $\beta$ in (74) satisfies mse $_{Y_{i}} \leq \beta<$ mse $_{\infty}$. Later on in Section IV-F we will show that this condition is indeed satisfied by the optimal solution to 64.

In order to solve (74), we first find a candidate solution to (74) by solving a free boundary problem; then we prove that the free boundary solution is indeed the value function of (74):

1) A Candidate Solution to (74): Now, we show how to solve (74). The general optimal stopping theory in Chapter I of [12] tells us that the following guess of the stopping time should be optimal for Problem (74):

$$
\tau_{*}=\inf \left\{t \geq 0:\left|V_{t}\right| \geq v_{*}\right\},
$$

where $v_{*} \geq 0$ is the optimal stopping threshold to be found. Observe that in this guess, the continuation region $\left(-v_{*}, v_{*}\right)$ is assumed symmetric around zero. This is because the OU process is symmetric, i.e., the process $\left\{-V_{t}, t \geq 0\right\}$ is also an $\mathrm{OU}$ process started at $-V_{0}=-v$. Similarly, we can also argue that the value function of problem (74) should be even.

According to [12, Chapter 8], and [13, Chapter 10], the value function and the optimal stopping threshold $v_{*}$ should satisfy the following free boundary problem:

$$
\begin{aligned}
& \frac{\sigma^{2}}{2} H^{\prime \prime}(v)-\theta v H^{\prime}(v)=v^{2}-\beta, \quad v \in\left(-v_{*}, v_{*}\right), \\
& H\left( \pm v_{*}\right)=-\gamma v_{*}^{2}, \\
& H^{\prime}\left( \pm v_{*}\right)=\mp 2 \gamma v_{*} .
\end{aligned}
$$

In Appendix $\mathrm{K}$, we use the integrating factor method [63, Sec. I.5] to find the general solution to (76), which is given by

$$
\begin{aligned}
H(v)= & -\frac{v^{2}}{2 \theta}+\left(\frac{1}{2 \theta}-\frac{\beta}{\sigma^{2}}\right){ }_{2} F_{2}\left(1,1 ; \frac{3}{2}, 2 ; \frac{\theta}{\sigma^{2}} v^{2}\right) v^{2} \\
& +C_{1} \operatorname{erfi}\left(\frac{\sqrt{\theta}}{\sigma} v\right)+C_{2}, \quad v \in\left(-v_{*}, v_{*}\right),
\end{aligned}
$$

where $C_{1}$ and $C_{2}$ are constants to be found for satisfying 
(77)-(78), and erfi $(x)$ is the imaginary error function, i.e.,

$$
\operatorname{erfi}(x)=\frac{2}{\sqrt{\pi}} \int_{0}^{x} e^{t^{2}} d t .
$$

Because $H(v)$ should be even but erfi $(x)$ is odd, we should choose $C_{1}=0$. Further, in order to satisfy the boundary condition (77), $C_{2}$ is chosen as

$$
C_{2}=\frac{1}{2 \theta} \mathbb{E}\left(e^{-2 \theta Y_{i}}\right) v_{*}^{2}-\left(\frac{1}{2 \theta}-\frac{\beta}{\sigma^{2}}\right){ }_{2} F_{2}\left(1,1 ; \frac{3}{2}, 2 ; \frac{\theta}{\sigma^{2}} v_{*}^{2}\right) v_{*}^{2},
$$

where we have used (24). With this, the expression of $H(v)$ is obtained in the continuation region $\left(-v_{*}, v_{*}\right)$. In the stopping region $|v| \geq v_{*}$, the stopping time in (75) is simply $\tau_{*}=0$, because $\left|V_{0}\right|=|v| \geq v_{*}$. Hence, if $|v| \geq v_{*}$, the objective value achieved by the sampling time (75) is

$$
\mathbb{E}_{v}\left[-\gamma v^{2}-\int_{0}^{0}\left(V_{s}^{2}-\beta\right) d s\right]=-\gamma v^{2} .
$$

Combining (79)-(82), we obtain a candidate of the value function for (74):

$$
H(v)=\left\{\begin{array}{lr}
-\frac{v^{2}}{2 \theta}+\left(\frac{1}{2 \theta}-\frac{\beta}{\sigma^{2}}\right){ }_{2} F_{2}\left(1,1 ; \frac{3}{2}, 2 ; \frac{\theta}{\sigma^{2}} v^{2}\right) v^{2}+C_{2}, \\
-\gamma v^{2}, & \text { if }|v|<v_{*}, \\
& \text { if }|v| \geq v_{*} .
\end{array}\right.
$$

Next, we find a candidate value of the optimal stopping threshold $v_{*}$. By taking the gradient of $H(v)$, we get

$H^{\prime}(v)=-\frac{v}{\theta}+\left(\frac{\sigma}{\theta^{\frac{3}{2}}}-\frac{2 \beta}{\sigma \sqrt{\theta}}\right) F\left(\frac{\sqrt{\theta}}{\sigma} v\right), \quad v \in\left(-v_{*}, v_{*}\right)$,

where

$$
F(x)=e^{x^{2}} \int_{0}^{x} e^{-t^{2}} d t .
$$

The boundary condition (78) implies that $v_{*}$ is the root of

$$
-\frac{v}{\theta}+\left(\frac{\sigma}{\theta^{\frac{3}{2}}}-\frac{2 \beta}{\sigma \sqrt{\theta}}\right) F\left(\frac{\sqrt{\theta}}{\sigma} v\right)=-2 \gamma v .
$$

Substituting (14), (15), and (24) into (86), yields that $v_{*}$ is the root of

$$
\left(\mathrm{mse}_{\infty}-\beta\right) G\left(\frac{\sqrt{\theta}}{\sigma} v\right)=\mathrm{mse}_{\infty}-\mathrm{mse}_{Y_{i}},
$$

where $G(\cdot)$ is defined in (16). Because mse $_{Y_{i}} \leq \beta<$ mse $_{\infty}$, $G(x)$ is strictly increasing on $[0, \infty)$, and $G(0)=1$, we know that (87) has a unique non-negative root $v_{*}$. Further, the root $v_{*}$ can be expressed as a function $v(\beta)$ of $\beta$, where $v(\beta)$ is defined in (19). By this, we obtain a candidate solution to (74).

2) Verification of the Optimality of the Candidate Solution: Next, we use Itô's formula to verify the above candidate solution is indeed optimal, as stated in the following theorem:

Theorem 5. If mse $_{Y_{i}} \leq \beta<$ mse $_{\infty}$, then for all $v \in \mathbb{R}, H(v)$ in (83) is the value function of the optimal stopping problem (74). In addition, the optimal stopping time for solving (74) is $\tau_{*}$ in (75), where $v_{*}=v(\beta)$ is given by (19).
In order to prove Theorem 5, we need to establish the following properties of $H(v)$ in (83), for the case that mse $_{Y_{i}} \leq$ $\beta<$ mse $_{\infty}$ is satisfied in (74):

Lemma 9. $H(v)=\mathbb{E}_{v}\left[-\gamma V_{\tau_{*}}^{2}-\int_{0}^{\tau_{*}}\left(V_{s}^{2}-\beta\right) d s\right]$.

Proof. See Appendix L

Lemma 10. $H(v) \geq-\gamma v^{2}$ for all $v \in \mathbb{R}$.

Proof. See Appendix M.

A function $f(v)$ is said to be excessive for the process $V_{t}$ if

$$
\mathbb{E}_{v} f\left(V_{t}\right) \leq f(v), \forall t \geq 0, v \in \mathbb{R} .
$$

By using Itô's formula in stochastic calculus, we can obtain

Lemma 11. The function $H(v)$ is excessive for the process $V_{t}$.

Proof. See Appendix $\mathbf{N}$

Now, we are ready to prove Theorem 5

Proof of Theorem [5. In Lemmas 9,11, we have shown that $H(v)=\mathbb{E}_{v}\left[-\gamma V_{\tau_{*}}^{2}-\int_{0}^{\tau_{*}}\left(V_{s}^{2}-\beta\right) d s\right], H(v) \geq-\gamma v^{2}$, and $H(v)$ is an excessive function. Moreover, from the proof of Lemma 9 , we know that $\mathbb{E}_{v}\left[\tau_{*}\right]<\infty$ holds for all $v \in \mathbb{R}$. Hence, $\mathbb{P}_{v}\left(\tau_{*}<\infty\right)=1$ for all $v \in \mathbb{R}$. These conditions and Theorem 1.11 in [12, Section 1.2] imply that $\tau_{*}$ is an optimal stopping time of (74). This completes the proof.

Because (72) is a special case of (74), we can get from Theorem 5 that

Corollary 1. If mse $_{Y_{i}} \leq \beta<\mathrm{mse}_{\infty}$, then a solution to is $\left(Z_{1}(\beta), Z_{2}(\beta), \ldots\right)$, where

$$
Z_{i}(\beta)=\inf \left\{t \geq 0:\left|O_{Y_{i}+t}\right| \geq v(\beta)\right\},
$$

and $v(\beta)$ is defined in (19).

F. Zero Duality Gap between (64) and (68)

Strong duality is established in the following thoerem:

Theorem 6. If the service times $Y_{i}$ are i.i.d. with $0<\mathbb{E}\left[Y_{i}\right]<$ $\infty$, then the duality gap between (64) and (68) is zero. Further, $\left(Z_{0}(\beta), Z_{1}(\beta), \ldots\right)$ is an optimal solution to both (64) and (68), where $Z_{i}(\beta)$ is determined by

$$
Z_{i}(\beta)=\inf \left\{t \geq 0:\left|O_{Y_{i}+t}\right| \geq v(\beta)\right\},
$$

$v(\beta)$ is defined in (19), $\beta \geq 0$ is the root of

$$
\mathbb{E}\left[\int_{Y_{i}}^{Y_{i}+Z_{i}(\beta)+Y_{i+1}} O_{t}^{2} d t\right]-\beta \mathbb{E}\left[Y_{i}+Z_{i}(\beta)\right]=0,
$$

if $\mathbb{E}\left[Y_{i}+Z_{i}(\beta)\right]>1 / f_{\max }$; otherwise, $\beta$ is the root of $\mathbb{E}\left[Y_{i}+\right.$ $\left.Z_{i}(\beta)\right]=1 / f_{\max }$. In both cases, mse $_{Y_{i}} \leq \beta<\mathrm{mse}_{\infty}$ is satisfied, and hence (19) is well-defined. Further, the optimal objective value to (63) is given by

$$
\text { mse }_{\text {opt }}=\frac{\mathbb{E}\left[\int_{Y_{i}}^{Y_{i}+Z_{i}(\beta)+Y_{i+1}} O_{t}^{2} d t\right]}{\mathbb{E}\left[Y_{i}+Z_{i}(\beta)\right]} .
$$




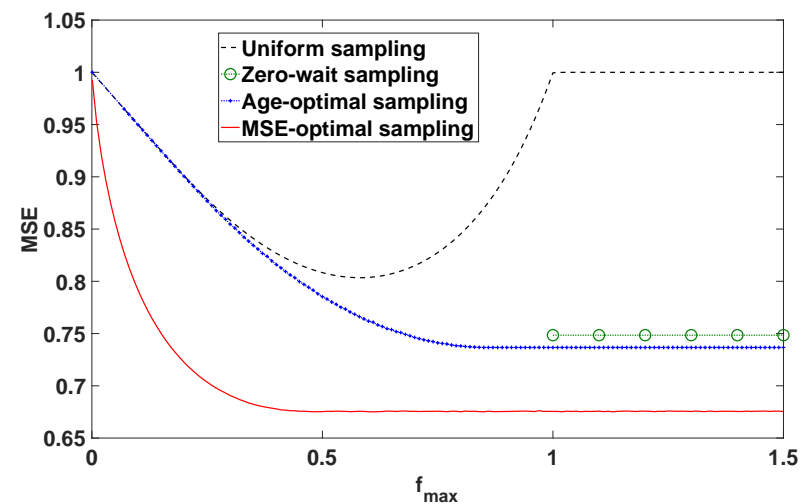

Fig. 8: MSE vs $f_{\max }$ tradeoff for i.i.d. exponential service time with $\mathbb{E}\left[Y_{i}\right]=1$, where the parameters of the OU process are $\sigma=1$ and $\theta=0.5$.

Proof. We use [60, Prop. 6.2.5] to find a geometric multiplier for (64). This suggests that the duality gap between (64) and (68) must be zero, because otherwise there exists no geometric multiplier [60, Prop. 6.2.3(b)]. The details are provided in Appendix 0

Hence, Theorem 2 follows from Theorem 6 Because Theorem 1 is a special case of Theorem 2 , Theorem 11 is also proven.

\section{Numerical COMPARISONS}

In this section, we evaluate the estimation error achieved by the following four sampling policies:

1. Uniform sampling: Periodic sampling with a period given by $S_{i+1}-S_{i}=1 / f_{\max }$.

2. Zero-wait sampling [1], [19]: The sampling policy given by

$$
S_{i+1}=S_{i}+Y_{i},
$$

which is infeasible when $f_{\max }<1 / \mathbb{E}\left[Y_{i}\right]$.

3. Age-optimal sampling [14]: The sampling policy given by Theorem 4

4. MSE-optimal sampling: The sampling policy given by Theorem 1

Let $\mathrm{mse}_{\text {uniform }}, \mathrm{mse}_{\text {zero-wait }}$, $\mathrm{mse}_{\text {age-opt }}$, and $\mathrm{mse}_{\text {opt }}$, be the MSEs of uniform sampling, zero-wait sampling, age-optimal sampling, MSE-optimal sampling, respectively. We can obtain

$$
\begin{aligned}
& \mathrm{mse}_{Y_{i}} \leq \mathrm{mse}_{\text {opt }} \leq \mathrm{mse}_{\text {age-opt }} \leq \mathrm{mse}_{\text {uniform }} \leq \mathrm{mse}_{\infty}, \\
& \mathrm{mse}_{\text {age-opt }} \leq \mathrm{mse}_{\text {zero-wait }} \leq \mathrm{mse}_{\infty},
\end{aligned}
$$

whenever zero-wait sampling is feasible, which fit with our numerical results. The expectations in (25) and (26) are evaluated by taking the average over 1 million samples. The parameters of the OU process are given by $\sigma=1, \theta=0.5$, and $\mu$ can be chosen arbitrarily because it does not affect the estimation error.

Figure 8 illustrates the tradeoff between the MSE and $f_{\max }$ for i.i.d. exponential service times with mean $\mathbb{E}\left[Y_{i}\right]=1$.

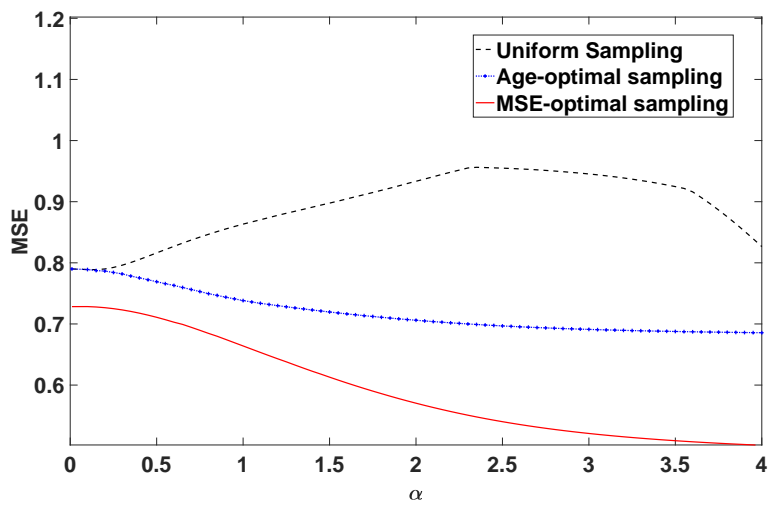

Fig. 9: MSE vs. the scale parameter $\alpha$ of i.i.d. normalized lognormal service time distribution with $\mathbb{E}\left[Y_{i}\right]=1$ and $f_{\max }=$ 0.8 , where the parameters of the OU process are $\sigma=1$ and $\theta=0.5$. Zero-wait sampling is not feasible here as $f_{\max }<$ $1 / \mathbb{E}\left[Y_{i}\right]$ and hence is not plotted.

Because $\mathbb{E}\left[Y_{i}\right]=1$, the maximum throughput of the queue is 1. The lower bound mse $_{Y_{i}}$ is 0.5 and the upper bound mse $_{\infty}$ is 1 . In fact, as $Y_{i}$ is an exponential random variable with mean $1, \frac{\sigma^{2}}{2 \theta}\left(1-e^{-2 \theta Y_{i}}\right)$ has a uniform distribution on $[0,1]$. Hence, mse $_{Y_{i}}=0.5$. For small values of $f_{\max }$, ageoptimal sampling is similar to uniform sampling, and hence mse $_{\text {age-opt }}$ and mse $_{\text {uniform }}$ are close to each other in the regime. However, as $f_{\max }$ grows, mse uniform reaches the upper bound mse $_{\infty}$ and remains constant for $f_{\max } \geq 1$. This is because the queue length of uniform sampling is large at high sampling frequencies. In particular, when $f_{\max } \geq 1$, the queue length of uniform sampling is infinite. On the other hand, mse age-opt and mse $_{\text {opt }}$ decrease with respect to $f_{\max }$. The reason behind this is that the set of feasible sampling policies satisfying the constraint in (11) and (48) becomes larger as $f_{\max }$ grows, and hence the optimal values of (11) and (48) are decreasing in

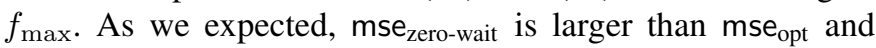
mse $_{\text {age-opt }}$. Moreover, all of them are between the lower bound mse $_{Y_{i}}$ and upper bound $\mathrm{mse}_{\infty}$.

Figures 9 and 10 depict the MSE of i.i.d. normalized log-normal service time for $f_{\max }=0.8$ and $f_{\max }=1.2$, respectively, where $Y_{i}=e^{\alpha X_{i}} / \mathbb{E}\left[e^{\alpha X_{i}}\right], \alpha>0$ is the scale parameter of log-normal distribution, and $\left(X_{1}, X_{2}, \ldots\right)$ are i.i.d. Gaussian random variables with zero mean and unit variance. Because $\mathbb{E}\left[Y_{i}\right]=1$, the maximum throughput of the queue is 1 . In Fig. 9 since $f_{\max }<1$, zero-wait sampling is not feasible and hence is not plotted. As the scale parameter $\alpha$ grows, the tail of the log-normal distribution becomes heavier.

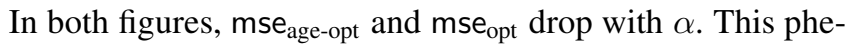
nomenon may look surprising at first sight, because mse age-opt $_{\text {a }}$ and $\mathrm{mse}_{\mathrm{opt}}$ grow quickly in $\alpha$ in the previous study [4] on the Wiener process. To understand this phenomenon, let us consider the age penalty function $p\left(\Delta_{t}\right)$ in (46) for the $\mathrm{OU}$ process. As the scale parameter $\alpha$ grows, the service time tends to become either shorter or much longer than the mean $\mathbb{E}\left[Y_{i}\right]$, rather than being close to $\mathbb{E}\left[Y_{i}\right]$. When $\Delta_{t}$ is small, $p\left(\Delta_{t}\right)$ reduces quickly in $\Delta_{t}$, and hence the service time smaller than $\mathbb{E}\left[Y_{i}\right]$ leads to a fast decrease in the average age penalty; 


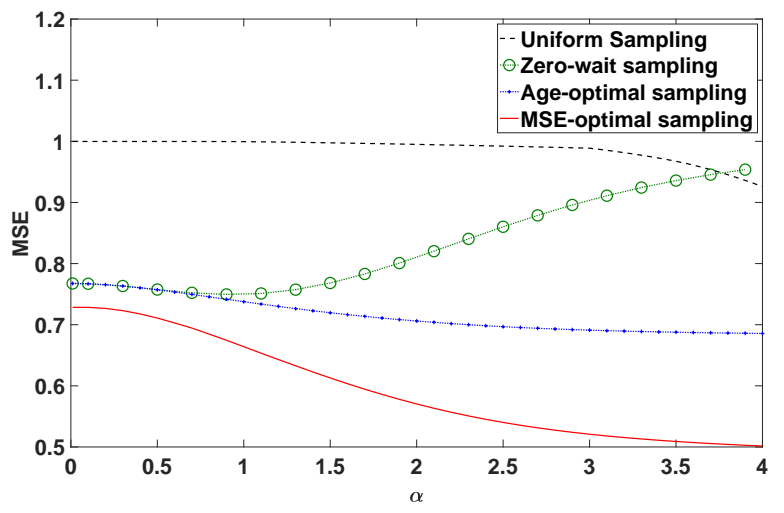

Fig. 10: MSE vs. the scale parameter $\alpha$ of i.i.d. normalized lognormal service time distribution $\mathbb{E}\left[Y_{i}\right]=1$ and $f_{\max }=1.2$, where the parameters of the OU process are $\sigma=1, \theta=0.5$.

when $\Delta_{t}$ is quite large, $p\left(\Delta_{t}\right)$ cannot increase much because it is upper bounded by mse $_{\infty}$, hence the service time much longer than $\mathbb{E}\left[Y_{i}\right]$ would not increase the average age penalty by much. By combining these two trends, the average age penalty mse age-opt decreases in $\alpha$. The dropping of $\mathrm{mse}_{\text {opt }}$ in $\alpha$ can be understood in a similar fashion. On the other hand, the age penalty function of the Wiener process is $p\left(\Delta_{t}\right)=\Delta_{t}$, which is quite different from the case considered here. We also observe that in both figures, the gap between mse $_{\text {opt }}$ and mse $_{\text {age-opt }}$ increases as $\alpha$ grows.

\section{CONCLUSION}

In this paper, we have studied the optimal sampler design for remote estimation of $\mathrm{OU}$ processes through queues. We have developed optimal causal sampling policies that minimize the estimation error of $\mathrm{OU}$ processes subject to a sampling rate constraint. These optimal sampling policies have nice structures and are easy to compute. A connection between remote estimation and nonlinear age metrics has been found. The structural properties of the optimal sampling policies shed lights on the possible structure of the optimal sampler designs for more general signal models, such as Feller processes, which is an important future research direction.

\section{ACKNOWLEDGEMENT}

The authors are grateful to Thaddeus Roppel for a suggestion on this work.

\section{REFERENCES}

[1] S. Kaul, R. D. Yates, and M. Gruteser, "Real-time status: How often should one update?" in IEEE INFOCOM, 2012.

[2] X. Song and J. W. S. Liu, "Performance of multiversion concurrency control algorithms in maintaining temporal consistency," in Proceedings., Fourteenth Annual International Computer Software and Applications Conference, Oct 1990, pp. 132-139.

[3] E. Altman, R. El-Azouzi, D. S. Menasche, and Y. Xu, "Forever young: Aging control for hybrid networks," in ACM MobiHoc, 2019.

[4] Y. Sun, Y. Polyanskiy, and E. Uysal, "Sampling of the Wiener process for remote estimation over a channel with random delay," IEEE Trans. Inf. Theory, vol. 66, no. 2, pp. 1118-1135, Feb 2020.

[5] G. E. Uhlenbeck and L. S. Ornstein, "On the theory of the Brownian motion," Phys. Rev., vol. 36, pp. 823-841, Sept. 1930.
[6] J. L. Doob, "The Brownian movement and stochastic equations," Annals of Mathematics, vol. 43, no. 2, pp. 351-369, 1942.

[7] L. Evans, S. Keef, and J. Okunev, "Modelling real interest rates," Journal of Banking and Finance, vol. 18, no. 1, pp. 153 - 165, 1994.

[8] A. Cika, M. Badiu, and J. Coon, "Quantifying link stability in ad hoc wireless networks subject to Ornstein-Uhlenbeck mobility," in IEEE ICC, 2019.

[9] H. Kim, J. Park, M. Bennis, and S. Kim, "Massive UAV-to-ground communication and its stable movement control: A mean-field approach," in IEEE SPAWC, June 2018, pp. 1-5.

[10] G. M. Lipsa and N. C. Martins, "Remote state estimation with communication costs for first-order LTI systems," IEEE Trans. Auto. Control, vol. 56, no. 9, pp. 2013-2025, Sept. 2011.

[11] E. Vinogradov, H. Sallouha, S. D. Bast, M. M. Azari, and S. Pollin, "Tutorial on uavs: A blue sky view onwireless communication," Journal of Mobile Multimedia, vol. 14, no. 4, p. 395-468, 2018.

[12] G. Peskir and A. N. Shiryaev, Optimal Stopping and Free-Boundary Problems. Basel, Switzerland: Birkhäuswer Verlag, 2006.

[13] B. Øksendal, Stochastic Differential Equations: An Introduction with Applications, 5th ed. Springer-Verlag Berlin Heidelberg, 2000.

[14] Y. Sun and B. Cyr, "Sampling for data freshness optimization: Nonlinear age functions," J. Commun. Netw., vol. 21, no. 3, pp. 204-219, 2019.

[15] —, "Information aging through queues: A mutual information perspective," in IEEE SPAWC Workshop, 2018.

[16] Y. Xiao and Y. Sun, "A dynamic jamming game for real-time status updates," in IEEE INFOCOM AoI Workshop, April 2018, pp. 354-360.

[17] A. M. Bedewy, Y. Sun, S. Kompella, and N. B. Shroff, "Age-optimal sampling and transmission scheduling in multi-source systems," in $A C M$ MobiHoc, 2019.

[18] C. Kam, S. Kompella, G. D. Nguyen, and A. Ephremides, "Effect of message transmission path diversity on status age," IEEE Trans. Inf. Theory, vol. 62, no. 3, pp. 1360-1374, Mar. 2016.

[19] Y. Sun, E. Uysal-Biyikoglu, R. D. Yates, C. E. Koksal, and N. B. Shroff, "Update or wait: How to keep your data fresh," IEEE Trans. Inf. Theory, vol. 63, no. 11, pp. 7492-7508, Nov. 2017.

[20] C. Kam, S. Kompella, G. D. Nguyen, J. E. Wieselthier, and A. Ephremides, "On the age of information with packet deadlines," IEEE Trans. Inf. Theory, vol. 64, no. 9, pp. 6419-6428, Sept. 2018.

[21] R. D. Yates and S. K. Kaul, "The age of information: Real-time status updating by multiple sources," IEEE Trans. Inf. Theory, vol. 65 , no. 3 , pp. 1807-1827, Mar. 2019.

[22] Q. He, D. Yuan, and A. Ephremides, "Optimal link scheduling for age minimization in wireless systems," IEEE Trans. Inf. Theory, vol. 64, no. 7, pp. 5381-5394, July 2018.

[23] C. Joo and A. Eryilmaz, "Wireless scheduling for information freshness and synchrony: Drift-based design and heavy-traffic analysis," IEEE/ACM Trans. Netw., vol. 26, no. 6, pp. 2556-2568, Dec 2018.

[24] A. M. Bedewy, Y. Sun, and N. B. Shroff, "Minimizing the age of the information through queues," IEEE Trans. Inf. Theory, vol. 65 , no. 8 , pp. 5215-5232, Aug 2019.

[25] 1 , "The age of information in multihop networks," IEEE/ACM Trans. Netw., vol. 27, no. 3, pp. 1248-1257, June 2019.

[26] Y. Sun, E. Uysal-Biyikoglu, and S. Kompella, "Age-optimal updates of multiple information flows," in IEEE INFOCOM AoI Workshop, 2018.

[27] I. Kadota, A. Sinha, and E. Modiano, "Optimizing age of information in wireless networks with throughput constraints," in IEEE INFOCOM, April 2018, pp. 1844-1852.

[28] R. Talak, S. Karaman, and E. Modiano, "Optimizing information freshness in wireless networks under general interference constraints," in ACM MobiHoc, 2018.

[29] N. Lu, B. Ji, and B. Li, "Age-based scheduling: Improving data freshness for wireless real-time traffic," in ACM MobiHoc, 2018.

[30] A. Maatouk, Y. Sun, A. Ephremides, and M. Assaad, "Status updates with priorities: Lexicographic optimality," in IEEE/IFIP WiOpt, 2020.

[31] B. Zhou and W. Saad, "Joint status sampling and updating for minimizing age of information in the Internet of things," IEEE Trans. Commun., vol. 67, no. 11, pp. 7468-7482, Nov 2019 .

[32] _ - "Minimum age of information in the Internet of things with nonuniform status packet sizes," IEEE Trans. Wireless Commun., vol. 19, no. 3, pp. 1933-1947, 2020.

[33] A. Kosta, N. Pappas, and V. Angelakis, Age of Information: A New Concept, Metric, and Tool. Now Publishers Inc, 2018.

[34] Y. Sun, I. Kadota, R. Talak, and E. Modiano, Age of Information: A New Metric for Information Freshness. Morgan \& Claypool, 2019. 
[35] A. M. Bedewy, Y. Sun, S. Kompella, and N. B. Shroff, "Optimal sampling and scheduling for timely status updates in multi-source networks," 2020, accepted by IEEE Trans. Inf. Theory.

[36] B. Hajek, K. Mitzel, and S. Yang, "Paging and registration in cellular networks: Jointly optimal policies and an iterative algorithm," IEEE Trans. Inf. Theory, vol. 54, no. 2, pp. 608-622, Feb 2008.

[37] M. Rabi, G. V. Moustakides, and J. S. Baras, "Adaptive sampling for linear state estimation," SIAM Journal on Control and Optimization, vol. 50, no. 2, pp. 672-702, 2012.

[38] A. Nayyar, T. Başar, D. Teneketzis, and V. V. Veeravalli, "Optimal strategies for communication and remote estimation with an energy harvesting sensor," IEEE Trans. Auto. Control, vol. 58, no. 9, pp. 22462260, Sept. 2013.

[39] K. Nar and T. Başar, "Sampling multidimensional Wiener processes," in IEEE CDC, Dec. 2014, pp. 3426-3431.

[40] X. Gao, E. Akyol, and T. Başar, "Optimal communication scheduling and remote estimation over an additive noise channel," Automatica, vol. 88 , pp. $57-69,2018$.

[41] J. Chakravorty and A. Mahajan, "Remote estimation over a packet-drop channel with Markovian state," IEEE Trans. Auto. Control, vol. 65, no. 5 , pp. 2016-2031, 2020.

[42] C.-H. Tsai and C.-C. Wang, "Unifying AoI minimization and remote estimation: Optimal sensor/controller coordination with random two-way delay," in IEEE INFOCOM, 2020.

[43] N. Guo and V. Kostina, "Optimal causal rate-constrained sampling for a class of continuous Markov processes," in IEEE ISIT, 2020.

[44] V. Jog, R. J. La, and N. C. Martins, "Channels, learning, queueing and remote estimation systems with a utilization-dependent component," 2019, coRR, abs/1905.04362.

[45] R. Durrett, Probability: Theory and Examples, 4th ed. Cambridge Univerisity Press, 2010.

[46] P. J. Haas, Stochastic Petri Nets: Modelling, Stability, Simulation. New York, NY: Springer New York, 2002.

[47] S. M. Ross, Applied Probability Models with Optimization Applications. San Francisco, CA: Holden-Day, 1970.

[48] H. Mine and S. Osaki, Markovian Decision Processes. New York: Elsevier, 1970.

[49] D. Hayman and M. Sobel, Stochastic models in Operations Research, Volume II: Stochastic Optimizations. New York: McGraw-Hill, 1984.

[50] E. A. Feinberg, "Constrained semi-Markov decision processes with average rewards," Zeitschrift für Operations Research, vol. 39, no. 3, pp. 257-288, 1994

[51] D. P. Bertsekas, Dynamic Programming and Optimal Control, 3rd ed. Belmont, MA: Athena Scientific, 2005, vol. 1.

[52] R. A. Maller, G. Müller, and A. Szimayer, "Ornstein-Uhlenbeck processes and extensions," in Handbook of Financial Time Series, T. Mikosch, J.-P. Kreiß, R. A. Davis, and T. G. Andersen, Eds. Berlin, Heidelberg: Springer Berlin Heidelberg, 2009, pp. 421-437.

[53] T. Soleymani, S. Hirche, and J. S. Baras, "Optimal information control in cyber-physical systems," IFAC-PapersOnLine, vol. 49, no. 22, pp. 1 $-6,2016$.

[54] I. Gradshteyn and I. Ryzhik, Table of Integrals, Series, and Products, 7th ed. Academic Press, 2007.

[55] F. W. Olver, D. W. Lozier, R. F. Boisvert, and C. W. Clark, NIST Handbook of Mathematical Functions. Cambridge University Press, 2010.

[56] J. H. Mathews and K. K. Fink, Numerical Methods Using Matlab. Simon \& Schuster, Inc., 1998.

[57] C.-H. Tsai and C.-C. Wang, "Age-of-information revisited: Two-way delay and distribution-oblivious online algorithm," in IEEE ISIT, 2020.

[58] M. Spivak, Calculus, 4th ed. Publish or Perish, 2008.

[59] W. Dinkelbach, "On nonlinear fractional programming," Management Science, vol. 13, no. 7, pp. 492-498, 1967.

[60] D. P. Bertsekas, A. Nedić, and A. E. Ozdaglar, Convex Analysis and Optimization. Belmont, MA: Athena Scientific, 2003.

[61] A. N. Borodin and P. Salminen, Handbook of Brownian Motion - Facts and Formulae. Basel, Switzerland: Birkhäuswer Verlag, 1996.

[62] S. M. Ross, Stochastic Processes, 2nd ed. John Wiley\& Sons, Inc., 1996.

[63] H. Amann, Ordinary Differential Equations An Introduction to Nonlinear Analysis. Berlin: Walter De Gruyter, 1990.

[64] G. Strang, Calculus. Wellesley-Cambridge Press, 1991.

[65] T. M. Liggett, Continuous Time Markov Processes: An Introduction. Providence, Rhode Island: American Mathematical Society, 2010.

[66] C. Jia and G. Zhao, "Moderate maximal inequalities for the OrnsteinUhlenbeck process," Proc. Amer. Math. Soc., vol. 148, pp. 3607-3615, 2020.
[67] H. V. Poor, An Introduction to Signal Detection and Estimation, 2nd ed New York, NY, USA: Springer-Verlag New York, Inc., 1994.

[68] A. Jeffrey and H.-H. Dai, Handbook of Mathematical Formulas and Integrals. Academic Press, 1995

[69] P. Morters and Y. Peres, Brownian Motion. Cambridge Univerisity Press, 2010.

\section{APPENDIX A \\ PROOF OF LEMMA 2}

Part (i): According to (66) and (69), the Lagrangian $L(\pi, \beta)$ is linear and strictly decreasing in $\beta$. Further, 677) tells us that $f(\beta)$ is the infimum of $L(\pi, \beta)$ among all policies $\pi \in \Pi_{1}$. Because the infimum of a linear and strictly decreasing function is concave and strictly decreasing, $f(\beta)$ is concave and strictly decreasing in $\beta$. Moreover, because $f(\beta)$ is concave, it is also continuous.

Part (ii): We first show that $f\left(\right.$ mse $\left._{Y_{i}}\right)>0$. According to (19), $v\left(\mathrm{mse}_{Y_{i}}\right)=0$. This, together with (25), (26), and (30), implies

$$
\begin{aligned}
f\left(\mathrm{mse}_{Y_{i}}\right) & =f_{1}\left(\mathrm{mse}_{Y_{i}}\right)-\mathrm{mse}_{Y_{i}} f_{2}\left(\mathrm{mse}_{Y_{i}}\right) \\
& =\operatorname{mse}_{\infty}\left\{\mathbb{E}\left[Y_{i}\right]-\gamma\right\}+\mathbb{E}\left[O_{Y_{i}}^{2}\right] \gamma-\operatorname{mse}_{Y_{i}} \mathbb{E}\left[Y_{i}\right] \\
& =\frac{\sigma^{2}}{2 \theta}\left\{\mathbb{E}\left[Y_{i}\right]-\frac{1}{2 \theta} \mathbb{E}\left[1-e^{-2 \theta Y_{i}}\right]\right. \\
& \left.+\frac{1}{2 \theta}\left\{\mathbb{E}\left[1-e^{-2 \theta Y_{i}}\right]\right\}^{2}-\mathbb{E}\left[1-e^{-2 \theta Y_{i}}\right] \mathbb{E}\left[Y_{i}\right]\right\} .
\end{aligned}
$$

Therefore, it suffices to prove that

$$
\begin{aligned}
& \mathbb{E}\left[Y_{i}\right]-\frac{1}{2 \theta} \mathbb{E}\left[1-e^{-2 \theta Y_{i}}\right]+\frac{1}{2 \theta}\left\{\mathbb{E}\left[1-e^{-2 \theta Y_{i}}\right]\right\}^{2} \\
& -\mathbb{E}\left[1-e^{-2 \theta Y_{i}}\right] \mathbb{E}\left[Y_{i}\right]>0,
\end{aligned}
$$

which can be simplified as

$$
\left(\mathbb{E}\left[Y_{i}\right]-\frac{1}{2 \theta} \mathbb{E}\left[1-e^{-2 \theta Y_{i}}\right]\right) \mathbb{E}\left[e^{-2 \theta Y_{i}}\right]>0 .
$$

Because $x>1-e^{-x}$ for all $x>0$ and $\mathbb{E}\left[Y_{i}\right]>0$, we get

$$
\mathbb{E}\left[2 \theta Y_{i}\right]>\mathbb{E}\left[1-e^{-2 \theta Y_{i}}\right] .
$$

By this, $f\left(\operatorname{mse}_{Y_{i}}\right)>0$ is proven.

Finally, we prove that $f\left(\mathrm{mse}_{\infty}\right)<0$. When $\beta \rightarrow \mathrm{mse}_{\infty}^{-}$, (19) tells us that $v(\beta)$ grows to infinite. Further, according to (25) and (26), $R_{1}(v(\beta))$ and $R_{2}(v(\beta))$ are quite large compared to $R_{1}\left(O_{Y_{i}}\right)$ and $R_{2}\left(O_{Y_{i}}\right)$. Therefore,

$$
\begin{aligned}
\lim _{\beta \rightarrow \mathrm{mse}_{\infty}^{-}} f(\beta) & =-\frac{1}{2 \theta} \lim _{\beta \rightarrow \mathrm{mse}_{\infty}^{-}} v^{2}(\beta) \mathbb{E}\left[e^{-2 \theta Y_{i}}\right]-\mathrm{mse}_{\infty} \gamma \\
& =-\infty .
\end{aligned}
$$

This completes the proof.

\section{APPENDIX B \\ PROOF OF LEMMA 3}

Part (i): From (22), it is evident that the function $f_{2}(\beta)$ is continuous and hence, from (40), $g(\beta)$ is also continuous. The 
derivatives of $R_{1}(v)$ in (25) and $v(\beta)$ in (19) are given by

$$
\begin{aligned}
& R_{1}^{\prime}(v)=\frac{\sqrt{\pi}}{\sigma \sqrt{\theta}} \operatorname{erf}\left(\frac{\sqrt{\theta}}{\sigma} v\right) e^{\frac{\theta}{\sigma^{2}} v^{2}}, \\
& v^{\prime}(\beta)=\frac{\sigma}{\sqrt{\theta}}\left\{G^{-1}\left(\frac{\mathrm{mse}_{\infty}-\mathrm{mse}_{Y_{i}}}{\mathrm{mse}_{\infty}-\beta}\right)\right\}^{\prime} .
\end{aligned}
$$

Denote

$$
G^{-1}\left(\frac{\mathrm{mse}_{\infty}-\mathrm{mse}_{Y_{i}}}{\mathrm{mse}_{\infty}-\beta}\right)=y .
$$

Then, by using the derivative of inverse function [64], $v^{\prime}(\beta)$ in (101) becomes

$$
v^{\prime}(\beta)=\frac{\sigma}{\sqrt{\theta}} \frac{1}{G^{\prime}(y)} \frac{\mathrm{mse}_{\infty}-\mathrm{mse}_{Y_{i}}}{\left(\mathrm{mse}_{\infty}-\beta\right)^{2}},
$$

where

$$
G^{\prime}(x)=\sqrt{\pi} e^{x^{2}} \operatorname{erf}(x)-\frac{e^{x^{2}}}{x^{2}} \frac{\sqrt{\pi}}{2} \operatorname{erf}(x)+\frac{1}{x}>0
$$

for all $x>0$. Hence, $v(\beta)$ is strictly increasing in $\beta$. From (100), we know $R_{1}^{\prime}(v)>0$, i.e., $R_{1}(v)$ is strictly increasing in $v$. Therefore, $R_{1}(v(\beta))$ is strictly increasing in $\beta$. This further implies that in (22), $\max \left\{R_{1}(v(\beta))-R_{1}\left(O_{Y_{i}}\right), 0\right\}$ is strictly increasing in $\beta$. Therefore, $\mathbb{E}\left[\max \left\{R_{1}(v(\beta))-R_{1}\left(O_{Y_{i}}\right), 0\right\}\right]$ is also strictly increasing in $\beta$ and hence, $f_{2}(\beta)$ is strictly increasing in $\beta$. Then, by (40), $g(\beta)$ is strictly decreasing in $\beta$. This completes the proof.

Part (ii): We first show that $g\left(\right.$ mse $\left._{Y_{i}}\right) \geq 0$. If the root of (20) does not satisfy (37), then, let $\beta^{*}$ is the root of (40). Therefore, $g\left(\beta^{*}\right)=0$. As mse $_{Y_{i}} \leq \beta \leq$ mse $_{\infty}$ and from part (i), $g(\beta)$ is strictly decreasing in $\beta$, we get that

$$
g\left(\mathrm{mse}_{Y_{i}}\right) \geq g\left(\beta^{*}\right)=0 .
$$

Hence, $g\left(\operatorname{mse}_{Y_{i}}\right) \geq 0$.

Finally, as $\beta \rightarrow \mathrm{mse}_{\infty}^{-}$, because $v(\beta)$ grows to infinite, $R_{1}(v(\beta))$ becomes quite large compared to $R_{1}\left(O_{Y_{i}}\right)$. Hence,

$$
\begin{aligned}
\lim _{\beta \rightarrow \mathrm{mse}_{\infty}^{-}} g(\beta) & =\frac{1}{f_{\max }}-\lim _{\beta \rightarrow \mathrm{mse}_{\infty}^{-}} R_{1}(v(\beta)) \\
& =-\infty .
\end{aligned}
$$

This complete the proof.

\section{APPENDix C}

PROOF OF (9)

The MMSE estimator $\hat{X}_{t}$ can be expressed as

$$
\begin{aligned}
\hat{X}_{t} & =\mathbb{E}\left[X_{t} \mid M_{t}\right] \\
& =\mathbb{E}\left[X_{t} \mid\left\{\left(S_{j}, X_{S_{j}}, D_{j}\right): D_{j} \leq t\right\}\right] .
\end{aligned}
$$

Recall that $U_{t}=\max \left\{S_{i}: D_{i} \leq t\right\}$ is the generation time of the latest received sample at time $t$. According to the strong Markov property of $X_{t}$ [12, Eq. (4.3.27)] and the fact that the $Y_{i}$ 's are independent of $\left\{X_{t}, t \geq 0\right\},\left\{U_{t}, X_{U_{t}}\right\}$ is a sufficient statistic for estimating $X_{t}$ based on $\left\{\left(S_{j}, X_{S_{j}}, D_{j}\right): D_{j} \leq t\right\}$. If $t \in\left[D_{i}, D_{i+1}\right)$, (4) suggests that $U_{t}=S_{i}$ and $X_{U_{t}}=X_{S_{i}}$.
This and (8) tell us that, if $t \in\left[D_{i}, D_{i+1}\right)$, then

$$
\begin{aligned}
\hat{X}_{t} & =\mathbb{E}\left[X_{t} \mid\left\{\left(S_{i}, X_{S_{i}}, D_{i}\right): D_{i} \leq t\right\}\right] \\
& =\mathbb{E}\left[X_{t} \mid S_{i}, X_{S_{i}}\right] \\
& =X_{S_{i}} e^{-\theta\left(t-S_{i}\right)}+\mu\left[1-e^{-\theta\left(t-S_{i}\right)}\right] .
\end{aligned}
$$

This completes the proof.

\section{APPENDIX D \\ PROOF OF LEMMA 4}

In any signal-ignorant policy, because the sampling times $S_{i}$ and the service times $Y_{i}$ are both independent of $\left\{X_{t}, t \geq 0\right\}$, the delivery times $D_{i}$ are also independent of $\left\{X_{t}, t \geq 0\right\}$. Hence, for any $t \in\left[D_{i}, D_{i+1}\right)$,

$$
\begin{aligned}
& \mathbb{E}\left[\left(X_{t}-\hat{X}_{t}\right)^{2} \mid S_{i}, D_{i}, D_{i+1}\right] \\
\stackrel{(a)}{=} & \mathbb{E}\left[\frac{\sigma^{2}}{2 \theta} e^{-2 \theta\left(t-S_{i}\right)} W_{e^{2 \theta\left(t-S_{i}\right)}-1}^{2} \mid S_{i}, D_{i}, D_{i+1}\right] \\
\stackrel{(b)}{=} & \frac{\sigma^{2}}{2 \theta}\left[1-e^{-2 \theta\left(t-S_{i}\right)}\right],
\end{aligned}
$$

where Step (a) is due to (8)-(9) and Step (b) is due to $\mathbb{E}\left[W_{t}^{2}\right]=$ $t$ for all constant $t \geq 0$. We note that in signal-aware sampling policies,

$$
\left(X_{t}-\hat{X}_{t}\right)^{2}=\frac{\sigma^{2}}{2 \theta} e^{-2 \theta\left(t-S_{i}\right)} W_{e^{2 \theta\left(t-S_{i}\right)}-1}^{2}
$$

could be correlated with $\left(S_{i}, D_{i}, D_{i+1}\right)$ and hence Step (b) of (109) may not hold. Substituting (4) into (109), yields that for all $t \geq 0$

$$
\mathbb{E}\left[\left(X_{t}-\hat{X}_{t}\right)^{2} \mid \pi, Y_{1}, Y_{2}, \ldots\right]=\frac{\sigma^{2}}{2 \theta}\left(1-e^{-2 \theta \Delta_{t}}\right),
$$

which is strictly increasing in $\Delta_{t}$. This completes the proof.

\section{APPENDIX E \\ PROOF OF (42)}

When $\sigma=1$, 877 can be expressed as

$$
(1-2 \theta \beta) G(\sqrt{\theta} v)=\mathbb{E}\left[e^{-2 \theta Y_{i}}\right],
$$

The error function $\operatorname{erf}(x)$ has a Maclaurin series representation, given by

$$
\operatorname{erf}(x)=\frac{2}{\sqrt{\pi}}\left[x-\frac{x^{3}}{3}+o\left(x^{3}\right)\right] .
$$

Hence, the Maclaurin series representation of $G(x)$ in (16) is

$$
G(x)=1+\frac{2 x^{2}}{3}+o\left(x^{2}\right) .
$$

Let $x=\sqrt{\theta} v$, we get

$$
G(\sqrt{\theta} v)=1+\frac{2}{3} \theta v^{2}+o(\theta) .
$$

In addition,

$$
\mathbb{E}\left[e^{-2 \theta Y_{i}}\right]=1-2 \theta \mathbb{E}\left[Y_{i}\right]+o(\theta) .
$$


Hence, (112) can be expressed as

$$
(1-2 \beta \theta)\left[1+\frac{2}{3} \theta v^{2}+o(\theta)\right]=1-2 \theta \mathbb{E}\left[Y_{i}\right]+o(\theta) .
$$

Expanding (117), yields

$$
2 \theta \mathbb{E}\left[Y_{i}\right]-2 \beta \theta+\frac{2}{3} \theta v^{2}+o(\theta)=0
$$

Dividing by $\theta$ and letting $\theta \rightarrow 0$ on both sides of (118), yields

$$
v^{2}-3\left(\beta-\mathbb{E}\left[Y_{i}\right]\right)=0 .
$$

Equation (119) has two roots $-\sqrt{3\left(\beta-\mathbb{E}\left[Y_{i}\right]\right)}$, and $\sqrt{3\left(\beta-\mathbb{E}\left[Y_{i}\right]\right)}$. If $v_{*}=-\sqrt{3\left(\beta-\mathbb{E}\left[Y_{i}\right]\right)}$, the free boundary problem in (76)-(78) are invalid. Hence, as $\theta \rightarrow 0$ and $\sigma=1$, the root of $[19)$ is $v_{*}=\sqrt{3\left(\beta-\mathbb{E}\left[Y_{i}\right]\right)}$. This completes the proof.

\section{APPENDIX F}

\section{PROOF OF LEMMA 5}

We first prove (56). It is known that the OU process $O_{t}$ is a Feller process [65, Section 5.5]. By using a property of Feller process in [65, Theorem 3.32], we get that

$$
\begin{aligned}
& O_{t}^{2}-\int_{0}^{t} \mathcal{G}\left(O_{s}^{2}\right) d s \\
= & O_{t}^{2}-\int_{0}^{t}\left(-\theta O_{s} 2 O_{s}+\sigma^{2}\right) d s \\
= & O_{t}^{2}-\sigma^{2} t+2 \theta \int_{0}^{t} O_{s}^{2} d s
\end{aligned}
$$

is a martingale, where $\mathcal{G}$ is the infinitesimal generator of $O_{t}$ defined in (55). According to [45], the minimum of two stopping times is a stopping time and constant times are stopping times. Hence, $t \wedge \tau$ is a bounded stopping time for every $t \in[0, \infty)$, where $x \wedge y=\min \{x, y\}$. Then, by Theorem 8.5.1 of [45], for every $t \in[0, \infty)$

$$
\mathbb{E}\left[\int_{0}^{t \wedge \tau} O_{s}^{2} d s\right]=\mathbb{E}\left[\frac{\sigma^{2}}{2 \theta}(t \wedge \tau)\right]-\mathbb{E}\left[\frac{1}{2 \theta} O_{t \wedge \tau}^{2}\right] .
$$

Because $\mathbb{E}\left[\int_{0}^{t \wedge \tau} O_{s}^{2} d s\right]$ and $\mathbb{E}[t \wedge \tau]$ are positive and increasing with respect to $t$, by using the monotone convergence theorem [45, Theorem 1.5.5], we get

$$
\begin{aligned}
\lim _{t \rightarrow \infty} \mathbb{E}\left[\int_{0}^{t \wedge \tau} O_{s}^{2} d s\right] & =\mathbb{E}\left[\int_{0}^{\tau} O_{s}^{2} d s\right], \\
\lim _{t \rightarrow \infty} \mathbb{E}[(t \wedge \tau)] & =\mathbb{E}[\tau] .
\end{aligned}
$$

In addition, according to [66, Theorem 2.2],

$$
\mathbb{E}\left[\max _{0 \leq s \leq \tau} O_{s}^{2}\right] \leq \frac{C}{\theta} \mathbb{E}\left[\log \left(1+\frac{\theta \tau}{\sigma}\right)\right] \leq \frac{C}{\sigma} \mathbb{E}[\tau]<\infty .
$$

Because $O_{t \wedge \tau}^{2} \leq \sup _{0 \leq s \leq \tau} O_{s}^{2}$ for all $t$ and $\sup _{0 \leq s \leq \tau} O_{s}^{2}$ is integratable, by invoking the dominated convergence theorem [45, Theorem 1.5.6], we have

$$
\lim _{t \rightarrow \infty} \mathbb{E}\left[O_{t \wedge \tau}^{2}\right]=\mathbb{E}\left[O_{\tau}^{2}\right] .
$$

Combining (122)-(125), (56) is proven.
We now prove (57) and (58). By using the solution of the ODE in Appendix $\mathrm{K}$, one can show that $R_{1}(v)$ in $(25)$ is the solution to the following ODE

$$
\frac{\sigma^{2}}{2} R_{1}^{\prime \prime}(v)-\theta v R_{1}^{\prime}(v)=1
$$

and $R_{2}(v)$ in (26) is the solution to the following ODE

$$
\frac{\sigma^{2}}{2} R_{2}^{\prime \prime}(v)-\theta v R_{2}^{\prime}(v)=v^{2} .
$$

In addition, $R_{1}(v)$ and $R_{2}(v)$ are twice continuously differentiable. According to Dynkin's formula in [13, Theorem 7.4.1 and the remark afterwards], because the initial value of $O_{t}$ is $O_{0}=0$, if $\tau$ is the first exit time of a bounded set, then

$$
\begin{aligned}
& \mathbb{E}_{0}\left[R_{1}\left(O_{\tau}\right)\right]=R_{1}(0)+\mathbb{E}_{0}\left[\int_{0}^{\tau} 1 d s\right]=R_{1}(0)+\mathbb{E}_{0}[\tau],(12 \\
& \mathbb{E}_{0}\left[R_{2}\left(O_{\tau}\right)\right]=R_{2}(0)+\mathbb{E}_{0}\left[\int_{0}^{\tau} O_{s}^{2} d s\right] .
\end{aligned}
$$

Because $R_{1}(0)=R_{2}(0)=0$, (57) and (58) follow. This completes the proof.

\section{APPENDIX G \\ PROOF OF LEMMA 6}

Suppose that in the sampling policy $\pi$, sample $i$ is generated when the server is busy sending another sample, and hence sample $i$ needs to wait for some time before being submitted to the server, i.e., $S_{i}<G_{i}$. Let us consider a virtual sampling policy $\pi^{\prime}=\left\{S_{0}, \ldots, S_{i-1}, G_{i}, S_{i+1}, \ldots\right\}$ such that the generation time of sample $i$ is postponed from $S_{i}$ to $G_{i}$. We call policy $\pi^{\prime}$ a virtual policy because it may happen that $G_{i}>$ $S_{i+1}$. However, this will not affect our proof below. We will show that the MSE of the sampling policy $\pi^{\prime}$ is smaller than that of the sampling policy $\pi=\left\{S_{0}, \ldots, S_{i-1}, S_{i}, S_{i+1}, \ldots\right\}$.

Note that $\left\{X_{t}: t \in[0, \infty)\right\}$ does not change according to the sampling policy, and the sample delivery times $\left\{D_{0}, D_{1}, D_{2}, \ldots\right\}$ remain the same in policy $\pi$ and policy $\pi^{\prime}$. Hence, the only difference between policies $\pi$ and $\pi^{\prime}$ is that the generation time of sample $i$ is postponed from $S_{i}$ to $G_{i}$. The MMSE estimator under policy $\pi$ is given by (9) and the MMSE estimator under policy $\pi^{\prime}$ is given by

$$
\begin{aligned}
\hat{X}_{t}^{\prime} & =\mathbb{E}\left[X_{t} \mid\left(S_{j}, X_{S_{j}}, D_{j}\right)_{j \leq i-1},\left(G_{i}, X_{G_{i}}, D_{i}\right)\right] \\
& = \begin{cases}\mathbb{E}\left[X_{t} \mid X_{G_{i}}, G_{i}\right], & t \in\left[D_{i}, D_{i+1}\right) ; \\
\mathbb{E}\left[X_{t} \mid X_{S_{j}}, S_{j}\right], & t \in\left[D_{j}, D_{j+1}\right), j \neq i .\end{cases}
\end{aligned}
$$

Next, we consider a third virtual sampling policy $\pi^{\prime \prime}$ in which the samples $\left(X_{G_{i}}, G_{i}\right)$ and $\left(X_{S_{i}}, S_{i}\right)$ are both delivered to the estimator at the same time $D_{i}$. Clearly, the estimator under policy $\pi^{\prime \prime}$ has more information than those under policies $\pi$ and $\pi^{\prime}$. By following the arguments in Appendix C] one can show that the MMSE estimator under policy $\pi^{\prime \prime}$ is

$$
\begin{aligned}
\hat{X}_{t}^{\prime \prime} & =\mathbb{E}\left[X_{t} \mid\left(S_{j}, X_{S_{j}}, D_{j}\right)_{j \leq i},\left(G_{i}, X_{G_{i}}, D_{i}\right)\right] \\
& = \begin{cases}\mathbb{E}\left[X_{t} \mid X_{G_{i}}, G_{i}\right], \quad t \in\left[D_{i}, D_{i+1}\right) ; \\
\mathbb{E}\left[X_{t} \mid X_{S_{j}}, S_{j}\right], \quad t \in\left[D_{j}, D_{j+1}\right), j \neq i .\end{cases}
\end{aligned}
$$

Notice that, because of the strong Markov property of OU process, the estimator under policy $\pi^{\prime \prime}$ uses the fresher sample 
$\left(X_{G_{i}}, G_{i}\right)$, instead of the stale sample $\left(X_{S_{i}}, S_{i}\right)$, to construct $\hat{X}_{t}^{\prime \prime}$ during $\left[D_{i}, D_{i+1}\right)$. Because the estimator under policy $\pi^{\prime \prime}$ has more information than that under policy $\pi$, one can imagine that policy $\pi^{\prime \prime}$ has a smaller estimation error than policy $\pi$, i.e.,

$$
\mathbb{E}\left\{\int_{D_{i}}^{D_{i+1}}\left(X_{t}-\hat{X}_{t}\right)^{2} d t\right\} \geq \mathbb{E}\left\{\int_{D_{i}}^{D_{i+1}}\left(X_{t}-\hat{X}_{t}^{\prime \prime}\right)^{2} d t\right\} .
$$

To prove 132, we invoke the orthogonality principle of the MMSE estimator [67, Prop. V.C.2] under policy $\pi^{\prime \prime}$ and obtain

$$
\mathbb{E}\left\{\int_{D_{i}}^{D_{i+1}} 2\left(X_{t}-\hat{X}_{t}^{\prime \prime}\right)\left(\hat{X}_{t}^{\prime \prime}-\hat{X}_{t}\right) d t\right\}=0,
$$

where we have used the fact that $\left(X_{G_{i}}, G_{i}\right)$ and $\left(X_{S_{i}}, S_{i}\right)$ are available by the MMSE estimator under policy $\pi^{\prime \prime}$. Next, from (133), we can get

$$
\begin{aligned}
& \mathbb{E}\left\{\int_{D_{i}}^{D_{i+1}}\left(X_{t}-\hat{X}_{t}\right)^{2} d t\right\} \\
= & \mathbb{E}\left\{\int_{D_{i}}^{D_{i+1}}\left(X_{t}-\hat{X}_{t}^{\prime \prime}\right)^{2}+\left(\hat{X}_{t}^{\prime \prime}-\hat{X}_{t}\right)^{2} d t\right\} \\
& +\mathbb{E}\left\{\int_{D_{i}}^{D_{i+1}} 2\left(X_{t}-\hat{X}_{t}^{\prime \prime}\right)\left(\hat{X}_{t}^{\prime \prime}-\hat{X}_{t}\right) d t\right\} \\
= & \mathbb{E}\left\{\int_{D_{i}}^{D_{i+1}}\left(X_{t}-\hat{X}_{t}^{\prime \prime}\right)^{2}+\left(\hat{X}_{t}^{\prime \prime}-\hat{X}_{t}\right)^{2} d t\right\} \\
\geq & \mathbb{E}\left\{\int_{D_{i}}^{D_{i+1}}\left(X_{t}-\hat{X}_{t}^{\prime \prime}\right)^{2} d t\right\} .
\end{aligned}
$$

In other words, the estimation error of policy $\pi^{\prime \prime}$ is no greater than that of policy $\pi$. Furthermore, by comparing (130) and (131), we can see that the MMSE estimators under policies $\pi^{\prime \prime}$ and $\pi^{\prime}$ are exact the same. Therefore, the estimation error of policy $\pi^{\prime}$ is no greater than that of policy $\pi$.

By repeating the above arguments for all samples $i$ satisfying $S_{i}<G_{i}$, one can show that the sampling policy $\left\{S_{0}, G_{1}, \ldots, G_{i-1}, G_{i}, G_{i+1}, \ldots\right\}$ is better than the sampling policy $\pi=\left\{S_{0}, S_{1}, \ldots, S_{i-1}, S_{i}, S_{i+1}, \ldots\right\}$. This completes the proof.

\section{APPENDIX H \\ PROOF OF (70)}

According to Lemma 5

$$
\begin{aligned}
& \mathbb{E}\left[\int_{Y_{i}+Z_{i}}^{Y_{i}+Z_{i}+Y_{i+1}} O_{s}^{2} d s\right] \\
= & \frac{\sigma^{2}}{2 \theta} \mathbb{E}\left[Y_{i+1}\right]-\frac{1}{2 \theta} \mathbb{E}\left[O_{Y_{i}+Z_{i}+Y_{i+1}}^{2}-O_{Y_{i}+Z_{i}}^{2}\right] .
\end{aligned}
$$

The second term in (135) can be expressed as

$$
\begin{aligned}
& \mathbb{E}\left[O_{Y_{i}+Z_{i}+Y_{i+1}}^{2}-O_{Y_{i}+Z_{i}}^{2}\right] \\
= & \mathbb{E}\left[\left(O_{Y_{i}+Z_{i}} e^{-\theta Y_{i+1}}+\frac{\sigma}{\sqrt{2 \theta}} e^{-\theta Y_{i+1}} W_{e^{2 \theta Y_{i+1}}-1}\right)^{2}\right. \\
= & \left.-O_{Y_{i}+Z_{i}}^{2}\right] \\
& +\mathbb{E}\left[O_{Y_{i}+Z_{i}}^{2}\left(e^{-2 \theta Y_{i+1}}-1\right)+\frac{\sigma^{2}}{2 \theta} e^{-2 \theta Y_{i+1}} W_{e^{2 \theta Y_{i+1}}-1}^{2}\right] \\
& \mathbb{E}\left[2 O_{Y_{i}+Z_{i}} e^{-\theta Y_{i+1}} \frac{\sigma}{\sqrt{2 \theta}} e^{-\theta Y_{i+1}} W_{e^{2 \theta Y_{i+1}}-1}\right] .
\end{aligned}
$$

Because $Y_{i+1}$ is independent of $O_{Y_{i}+Z_{i}}$ and $W_{t}$, we have $\mathbb{E}\left[O_{Y_{i}+Z_{i}}^{2}\left(e^{-2 \theta Y_{i+1}}-1\right)\right]=\mathbb{E}\left[O_{Y_{i}+Z_{i}}^{2}\right] \mathbb{E}\left[e^{-2 \theta Y_{i+1}}-1\right]$,

and

$$
\begin{aligned}
& \mathbb{E}\left[2 O_{Y_{i}+Z_{i}} e^{-\theta Y_{i+1}} \frac{\sigma}{\sqrt{2 \theta}} e^{-\theta Y_{i+1}} W_{e^{2 \theta Y_{i+1}}-1}\right] \\
= & \mathbb{E}\left[2 O_{Y_{i}+Z_{i}}\right] \mathbb{E}\left[e^{-\theta Y_{i+1}} \frac{\sigma}{\sqrt{2 \theta}} e^{-\theta Y_{i+1}} W_{e^{2 \theta Y_{i+1}}-1}\right] \\
\stackrel{(a)}{=} & \mathbb{E}\left[2 O_{Y_{i}+Z_{i}}\right] \mathbb{E}\left[\mathbb{E}\left[e^{-\theta Y_{i+1}} \frac{\sigma}{\sqrt{2 \theta}} e^{-\theta Y_{i+1}} W_{e^{2 \theta Y_{i+1}}-1} \mid Y_{i+1}\right]\right] .
\end{aligned}
$$

where Step (a) is due to the law of iterated expectations. Because $\mathbb{E}\left[W_{t}\right]=0$ for all constant $t \geq 0$, it holds for all realizations of $Y_{i+1}$ that

$$
\mathbb{E}\left[e^{-\theta Y_{i+1}} \frac{\sigma}{\sqrt{2 \theta}} e^{-\theta Y_{i+1}} W_{e^{2 \theta Y_{i+1}}-1} \mid Y_{i+1}\right]=0 .
$$

Hence,

$$
\mathbb{E}\left[2 O_{Y_{i}+Z_{i}} e^{-\theta Y_{i+1}} \frac{\sigma}{\sqrt{2 \theta}} e^{-\theta Y_{i+1}} W_{e^{2 \theta Y_{i+1}}-1}\right]=0 .
$$

In addition,

$$
\begin{aligned}
& \mathbb{E}\left[\frac{\sigma^{2}}{2 \theta} e^{-2 \theta Y_{i+1}} W_{e^{2 \theta Y_{i+1}}-1}^{2}\right] \\
\stackrel{(a)}{=} & \frac{\sigma^{2}}{2 \theta} \mathbb{E}\left[\mathbb{E}\left[e^{-2 \theta Y_{i+1}} W_{e^{2 \theta Y_{i+1}}-1}^{2} \mid Y_{i+1}\right]\right] \\
\stackrel{(b)}{=} & \frac{\sigma^{2}}{2 \theta} \mathbb{E}\left[1-e^{-2 \theta Y_{i+1}}\right],
\end{aligned}
$$

where Step (a) is due to the law of iterated expectations and Step (b) is due to $\mathbb{E}\left[W_{t}^{2}\right]=t$ for all constant $t \geq 0$. Hence,

$$
\begin{aligned}
& \mathbb{E}\left[\int_{Y_{i}+Z_{i}}^{Y_{i}+Z_{i}+Y_{i+1}} O_{s}^{2} d s\right] \\
= & \frac{\sigma^{2}}{2 \theta} \mathbb{E}\left[Y_{i+1}\right]+\gamma \mathbb{E}\left[O_{Y_{i}+Z_{i}}^{2}\right]-\frac{\sigma^{2}}{4 \theta^{2}} \mathbb{E}\left[1-e^{-2 \theta Y_{i+1}}\right] \\
= & \frac{\sigma^{2}}{2 \theta}\left[\mathbb{E}\left(Y_{i+1}\right)-\gamma\right]+\mathbb{E}\left[O_{Y_{i}+Z_{i}}^{2}\right] \gamma,
\end{aligned}
$$

where $\gamma$ is defined in (24). Using this, (70) can be shown readily. 


\section{APPENDIX I}

PROOF OF LEMMA 1

According to (8) and (9), the estimation error $\left(X_{t}-\hat{X}_{t}\right)$ is of the same distribution with $O_{t-S_{i}(\beta)}$ for $t \in\left[D_{i}(\beta), D_{i+1}(\beta)\right)$. We will use $\left(X_{t}-\hat{X}_{t}\right)$ and $O_{t-S_{i}(\beta)}$ interchangeably for $t \in$ $\left[D_{i}(\beta), D_{i+1}(\beta)\right)$. In order to prove Lemma 1 we need to consider the following two cases:

Case 1: If $\left|X_{D_{i}(\beta)}-\hat{X}_{D_{i}(\beta)}\right|=\left|O_{Y_{i}}\right| \geq v(\beta)$, then (18) tells us $S_{i+1}(\beta)=D_{i}(\beta)$. Hence,

$$
\begin{aligned}
D_{i}(\beta) & =S_{i}(\beta)+Y_{i}, \\
D_{i+1}(\beta) & =S_{i+1}(\beta)+Y_{i+1}=D_{i}(\beta)+Y_{i+1} .
\end{aligned}
$$

Using these and the fact that the $Y_{i}$ 's are independent of the OU process, we can obtain

$$
\mathbb{E}\left[D_{i+1}(\beta)-D_{i}(\beta)\left|O_{Y_{i}},\right| O_{Y_{i}} \mid \geq v(\beta)\right]=\mathbb{E}\left[Y_{i+1}\right],
$$

and

$$
\begin{aligned}
& \mathbb{E}\left[\int_{D_{i}(\beta)}^{D_{i+1}(\beta)}\left(X_{t}-\hat{X}_{t}\right)^{2} d t\left|O_{Y_{i}},\right| O_{Y_{i}} \mid \geq v(\beta)\right] \\
= & \mathbb{E}\left[\int_{Y_{i}}^{Y_{i}+Y_{i+1}} O_{s}^{2} d s\left|O_{Y_{i}},\right| O_{Y_{i}} \mid \geq v(\beta)\right] \\
\stackrel{(a)}{=} & \frac{\sigma^{2}}{2 \theta} \mathbb{E}\left[Y_{i+1}\right]+\gamma O_{Y_{i}}^{2}-\frac{\sigma^{2}}{4 \theta^{2}} \mathbb{E}\left[1-e^{-2 \theta Y_{i+1}}\right] \\
= & \operatorname{mse}_{\infty}\left[\mathbb{E}\left(Y_{i+1}\right)-\gamma\right]+O_{Y_{i}}^{2} \gamma,
\end{aligned}
$$

where Step (a) follows from the proof of (142).

Case 2: If $\left|X_{D_{i}(\beta)}-\hat{X}_{D_{i}(\beta)}\right|=\left|O_{Y_{i}}\right|<v(\beta)$, then (18) tells us that, almost surely,

$$
\left|X_{S_{i+1}(\beta)}-\hat{X}_{S_{i+1}(\beta)}\right|=v(\beta) .
$$

Let us consider the following equation:

$$
\begin{aligned}
& \mathbb{E}\left[D_{i+1}(\beta)-D_{i}(\beta)\left|O_{Y_{i}},\right| O_{Y_{i}} \mid<v(\beta)\right] \\
= & \mathbb{E}\left[\left(D_{i+1}(\beta)-S_{i+1}(\beta)\right)+\left(S_{i+1}(\beta)-S_{i}(\beta)\right)\right. \\
& \left.-\left(D_{i}(\beta)-S_{i}(\beta)\right)\left|O_{Y_{i}},\right| O_{Y_{i}} \mid<v(\beta)\right] .
\end{aligned}
$$

Because $D_{i+1}(\beta)=S_{i+1}(\beta)+Y_{i+1}$, the remaining task is to find $\mathbb{E}\left[S_{i+1}(\beta)-S_{i}(\beta)\left|O_{Y_{i}},\right| O_{Y_{i}} \mid<v(\beta)\right]$, and $\mathbb{E}\left[D_{i}(\beta)-S_{i}(\beta)\left[O_{Y_{i}},\left|O_{Y_{i}}\right|<v(\beta)\right]\right.$ to compute (148). By invoking Lemma 5 , we can obtain

$$
\begin{aligned}
& \mathbb{E}\left[S_{i+1}(\beta)-S_{i}(\beta)\left|O_{Y_{i}},\right| O_{Y_{i}} \mid<v(\beta)\right]=R_{1}(v(\beta)), \\
& \mathbb{E}\left[D_{i}(\beta)-S_{i}(\beta)\left|O_{Y_{i}},\right| O_{Y_{i}} \mid<v(\beta)\right]=R_{1}\left(\left|O_{Y_{i}}\right|\right),
\end{aligned}
$$

Substituting (149), (150), and $D_{i+1}(\beta)=S_{i+1}(\beta)+Y_{i+1}$ in (148), we get that

$$
\begin{aligned}
& \mathbb{E}\left[D_{i+1}(\beta)-D_{i}(\beta)\left|O_{Y_{i}},\right| O_{Y_{i}} \mid<v(\beta)\right] \\
= & \mathbb{E}\left[Y_{i+1}\right]+R_{1}(v(\beta))-R_{1}\left(\left|O_{Y_{i}}\right|\right) .
\end{aligned}
$$

In addition, let us consider the following equation:

$$
\begin{aligned}
& \mathbb{E}\left[\int_{D_{i}(\beta)}^{D_{i+1}(\beta)}\left(X_{t}-\hat{X}_{t}\right)^{2} d t\left|O_{Y_{i}},\right| O_{Y_{i}} \mid<v(\beta)\right] \\
&= \mathbb{E}\left[\int_{S_{i+1}(\beta)}^{D_{i+1}(\beta)}\left(X_{t}-\hat{X}_{t}\right)^{2} d t+\int_{S_{i}(\beta)}^{S_{i+1}(\beta)}\left(X_{t}-\hat{X}_{t}\right)^{2} d t\right. \\
&\left.\quad-\int_{S_{i}(\beta)}^{D_{i}(\beta)}\left(X_{t}-\hat{X}_{t}\right)^{2} d t\left|O_{Y_{i}},\right| O_{Y_{i}} \mid<v(\beta)\right]
\end{aligned}
$$

Next, we need to compute the expectations in (152). By invoking Lemma 5 again, we can obtain

$$
\begin{aligned}
& \mathbb{E}\left[\int_{S_{i}(\beta)}^{S_{i+1}(\beta)}\left(X_{t}-\hat{X}_{t}\right)^{2} d t\left|O_{Y_{i}},\right| O_{Y_{i}} \mid<v(\beta)\right] \\
= & \mathbb{E}\left[\int_{0}^{Y_{i}+Z_{i}} O_{s}^{2} d s\left|O_{Y_{i}},\right| O_{Y_{i}} \mid<v(\beta)\right]=R_{2}(v(\beta)), \\
& \mathbb{E}\left[\int_{S_{i}(\beta)}^{D_{i}(\beta)}\left(X_{t}-\hat{X}_{t}\right)^{2} d t\left|O_{Y_{i}},\right| O_{Y_{i}} \mid<v(\beta)\right] \\
= & \mathbb{E}\left[\int_{0}^{Y_{i}} O_{s}^{2} d s\left|O_{Y_{i}},\right| O_{Y_{i}} \mid<v(\beta)\right]=R_{2}\left(\left|O_{Y_{i}}\right|\right) .
\end{aligned}
$$

By substituting (153), (154), and (142) in (152), we have

$$
\begin{aligned}
& \mathbb{E}\left[\int_{D_{i}(\beta)}^{D_{i+1}(\beta)}\left(X_{t}-\hat{X}_{t}\right)^{2} d t\left|O_{Y_{i}},\right| O_{Y_{i}} \mid<v(\beta)\right] \\
= & \operatorname{mse}_{\infty}\left[\mathbb{E}\left(Y_{i+1}\right)-\gamma\right]+v^{2}(\beta) \gamma+R_{2}(v(\beta))-R_{2}\left(\left|O_{Y_{i}}\right|\right) .
\end{aligned}
$$

By combining (145) and (151) of the two cases, yields

$$
\begin{aligned}
& \mathbb{E}\left[D_{i+1}(\beta)-D_{i}(\beta) \mid O_{Y_{i}}\right] \\
= & \mathbb{E}\left[Y_{i+1}\right]+\max \left\{R_{1}(v(\beta))-R_{1}\left(\left|O_{Y_{i}}\right|\right), 0\right\} .
\end{aligned}
$$

Similarly, by combining (146) and 155) of the two cases, yields

$$
\begin{aligned}
& \mathbb{E}\left[\int_{D_{i}(\beta)}^{D_{i+1}(\beta)}\left(X_{t}-\hat{X}_{t}\right)^{2} d t \mid O_{Y_{i}}\right] \\
= & \operatorname{mse}_{\infty}\left[\mathbb{E}\left(Y_{i+1}\right)-\gamma\right]+\max \left\{v^{2}(\beta), O_{Y_{i}}^{2}\right\} \gamma \\
& +\max \left\{R_{2}(v(\beta))-R_{2}\left(\left|O_{Y_{i}}\right|\right), 0\right\} .
\end{aligned}
$$

Finally, by taking the expectation over $O_{Y_{i}}$ in (156) and (157) and using the fact that $R_{1}(\cdot)$ and $R_{2}(\cdot)$ are even functions, Lemma 1 is proven.

\section{APPENDIX J \\ PROOF OF LEMMA 8}

Because the $Y_{i}$ 's are i.i.d., (70) is determined by the control decision $Z_{i}$ and the information $\left(O_{Y_{i}}, Y_{i}\right)$. Hence, $\left(O_{Y_{i}}, Y_{i}\right)$ is a sufficient statistic for determining $Z_{i}$ in (67). Therefore, there exists an optimal policy $\left(Z_{0}, Z_{1}, \ldots\right)$ to (67), in which $Z_{i}$ is determined based on only $\left(O_{Y_{i}}, Y_{i}\right)$. By this, (67) is decomposed into a sequence of per-sample MDPs, given by (72). This completes the proof. 
APPENDIX K

PROOF OF (79)

Define $S(v)=H^{\prime}(v)$. Then, (76) becomes

$$
S^{\prime}(v)-\frac{2 \theta}{\sigma^{2}} v S(v)=\frac{2}{\sigma^{2}}\left(v^{2}-\beta\right) .
$$

Equation (158) can be solved by using the integrating factor method [63, Sec. I.5], which applies to any ODE of the form

$$
S^{\prime}(v)+a(v) S(v)=b(v) .
$$

In the case of (158),

$$
a(v)=-\frac{2 \theta}{\sigma^{2}} v, b(v)=\frac{2}{\sigma^{2}}\left(v^{2}-\beta\right) .
$$

The integrating factor of (158) is

$$
M(v)=e^{\int a(v) d v}=e^{-\frac{\theta}{\sigma^{2}} v^{2}} .
$$

Multiplying $e^{-\frac{\theta}{\sigma^{2}} v^{2}}$ on both sides of (158) and transforming the left-hand side into a total derivative, yields

$$
\left[S(v) e^{-\frac{\theta}{\sigma^{2}} v^{2}}\right]^{\prime}=b(v) e^{-\frac{\theta}{\sigma^{2}} v^{2}} .
$$

Taking the integration on both sides of (162), yields

$$
\begin{aligned}
S(v) e^{-\frac{\theta}{\sigma^{2}} v^{2}} & =\int \frac{2}{\sigma^{2}}\left(v^{2}-\beta\right) e^{-\frac{\theta}{\sigma^{2}} v^{2}} d v \\
& =\int \frac{2}{\sigma^{2}} e^{-\frac{\theta}{\sigma^{2}} v^{2}} v^{2} d v-\int \frac{2}{\sigma^{2}} \beta e^{-\frac{\theta}{\sigma^{2}} v^{2}} d v .
\end{aligned}
$$

The indefinite integrals in (163) are given by [68, Sec. 15.3.1, (Eq. 36)]

$$
\begin{aligned}
\int \frac{2}{\sigma^{2}} e^{-\frac{\theta}{\sigma^{2}} v^{2}} v^{2} d v & =\frac{\sqrt{\pi} \sigma}{2 \theta^{\frac{3}{2}}} \operatorname{erf}\left(\frac{\sqrt{\theta}}{\sigma} v\right)-\frac{v}{\theta} e^{-\frac{\theta}{\sigma^{2}} v^{2}}+C_{1} \\
\int \frac{2}{\sigma^{2}} \beta e^{-\frac{\theta}{\sigma^{2}} v^{2}} d v & =\frac{\sqrt{\pi} \beta}{\sigma \sqrt{\theta}} \operatorname{erf}\left(\frac{\sqrt{\theta}}{\sigma} v\right)+C_{2}
\end{aligned}
$$

where $\operatorname{erf}(\cdot)$ is the error function defined in (17). Combining (163)-(165), results in

$$
S(v)=\left(\frac{\sqrt{\pi} \sigma}{2 \theta^{\frac{3}{2}}}-\frac{\sqrt{\pi} \beta}{\sigma \sqrt{\theta}}\right) \operatorname{erf}\left(\frac{\sqrt{\theta}}{\sigma} v\right) e^{\frac{\theta}{\sigma^{2}} v^{2}}-\frac{v}{\theta}+C_{3} e^{\frac{\theta}{\sigma^{2}} v^{2}},
$$

where $C_{3}=C_{1}+C_{2}$. We need to integrate $S(v)$ in (166) again to get $H(v)$

$$
\begin{aligned}
H(v)= & \int S(v) d v \\
= & \int\left(\frac{\sqrt{\pi} \sigma}{2 \theta^{\frac{3}{2}}}-\frac{\sqrt{\pi} \beta}{\sigma \sqrt{\theta}}\right) \operatorname{erf}\left(\frac{\sqrt{\theta}}{\sigma} v\right) e^{\frac{\theta}{\sigma^{2}} v^{2}} d v-\int \frac{v}{\theta} d v \\
& +\int C_{3} e^{\frac{\theta}{\sigma^{2}} v^{2}} d v
\end{aligned}
$$

which requires the following integral [54, Sec. 8.250 (Eq. $1,4)]$ :

$$
\begin{aligned}
& \int \operatorname{erf}\left(\frac{\sqrt{\theta}}{\sigma} v\right) e^{\frac{\theta}{\sigma^{2}} v^{2}} d v \\
= & \frac{\sigma}{\sqrt{\theta} \sqrt{\pi}} \frac{\theta}{\sigma^{2}} v_{2}^{2} F_{2}\left(1,1 ; \frac{3}{2}, 2 ; \frac{\theta}{\sigma^{2}} v^{2}\right)+C .
\end{aligned}
$$

By using (168), we can compute the first integral of (167)

$$
\begin{aligned}
& \int\left(\frac{\sqrt{\pi} \sigma}{2 \theta^{\frac{3}{2}}}-\frac{\sqrt{\pi} \beta}{\sigma \sqrt{\theta}}\right) \operatorname{erf}\left(\frac{\sqrt{\theta}}{\sigma} v\right) e^{\frac{\theta}{\sigma^{2}} v^{2}} d v \\
= & \left(\frac{1}{2 \theta}-\frac{\beta}{\sigma^{2}}\right) v^{2}{ }_{2} F_{2}\left(1,1 ; \frac{3}{2}, 2 ; \frac{\theta}{\sigma^{2}} v^{2}\right)+C_{4} .
\end{aligned}
$$

The remaining integrals in (167) are as follows [54, Sec. 3.478 (Eq. 3)]

$$
\begin{aligned}
& \int C_{3} e^{\frac{\theta}{\sigma^{2}} v^{2}} d v=C_{5} \operatorname{erfi}\left(\frac{\sqrt{\theta}}{\sigma} v\right)+C_{6}, \\
& \int \frac{v}{\theta} d v=-\frac{v^{2}}{2 \theta}+C_{7},
\end{aligned}
$$

where $\operatorname{erfi}(\cdot)$ is the imaginary error function defined in $(80)$. Hence, by substituting (169), (170), and (171) in (167), $H(v)$ in (79) follows. This completes the proof of (79).

\section{APPENDIX L}

\section{Proof of LEMMa 9}

The proof of Lemma 9 consists of the following two cases: Case 1: If $|v| \geq v_{*}$, (75) implies $\tau_{*}=0$. Hence,

$$
\mathbb{E}_{v}\left[\tau_{*}|| v \mid \geq v_{*}\right]=\mathbb{E}_{v}\left[\int_{0}^{\tau_{*}} 1 d s|| v \mid \geq v_{*}\right]=0,
$$

and

$$
\mathbb{E}_{v}\left[\int_{0}^{\tau_{*}} V_{s}^{2} d s|| v \mid \geq v_{*}\right]=0 .
$$

Because $V_{0}=v$, we have

$$
\mathbb{E}_{v}\left[V_{\tau_{*}}^{2}\right]=\mathbb{E}_{v}\left[V_{0}^{2}\right]=v^{2} .
$$

By combining (172)-(174), we get

$$
\mathbb{E}_{v}\left[-\gamma V_{\tau_{*}}^{2}-\int_{0}^{\tau_{*}}\left(V_{s}^{2}-\beta\right) d s|| v \mid \geq v_{*}\right]=-\gamma v^{2} .
$$

Case 2: If $|v|<v_{*}$, (75) tells us that, almost surely,

$$
V_{\tau_{*}}=v_{*} .
$$

Similar to the proof of Lemma 1 , we can use Lemma 5 to obtain

$$
\begin{aligned}
& \mathbb{E}_{v}\left[\tau_{*}|| v \mid<v_{*}\right] \\
= & \mathbb{E}_{v}\left[\int_{0}^{\tau_{*}} 1 d s|| v \mid<v_{*}\right] \\
= & R_{1}\left(v_{*}\right)-R_{1}(v) \\
= & \frac{v_{*}^{2}}{\sigma^{2}}{ }_{2} F_{2}\left(1,1 ; \frac{3}{2}, 2 ; \frac{\theta}{\sigma^{2}} v_{*}^{2}\right)-\frac{v^{2}}{\sigma^{2}}{ }_{2} F_{2}\left(1,1 ; \frac{3}{2}, 2 ; \frac{\theta}{\sigma^{2}} v^{2}\right),
\end{aligned}
$$




$$
\begin{aligned}
& \mathbb{E}_{v}\left[\int_{0}^{\tau_{*}} V_{s}{ }^{2} d s|| v \mid<v_{*}\right] \\
= & R_{2}\left(v_{*}\right)-R_{2}(v) \\
= & -\frac{v_{*}^{2}}{2 \theta}+\frac{v_{*}^{2}}{2 \theta}{ }_{2} F_{2}\left(1,1 ; \frac{3}{2}, 2 ; \frac{\theta}{\sigma^{2}} v_{*}^{2}\right) \\
& +\frac{v^{2}}{2 \theta}-\frac{v^{2}}{2 \theta}{ }_{2} F_{2}\left(1,1 ; \frac{3}{2}, 2 ; \frac{\theta}{\sigma^{2}} v^{2}\right),
\end{aligned}
$$

and

$$
\mathbb{E}_{v}\left[V_{\tau_{*}}^{2}|| v \mid<v_{*}\right]=v_{*}^{2} .
$$

Combining (177)-(179), yields

$$
\begin{aligned}
& \mathbb{E}_{v}\left[-\gamma V_{\tau_{*}}^{2}-\int_{0}^{\tau_{*}}\left(V_{s}^{2}-\beta\right) d s|| v \mid<v_{*}\right] \\
= & -\frac{v^{2}}{2 \theta}+\left(\frac{1}{2 \theta}-\frac{\beta}{\sigma^{2}}\right){ }_{2} F_{2}\left(1,1 ; \frac{3}{2}, 2 ; \frac{\theta}{\sigma^{2}} v^{2}\right) v^{2} \\
& +\frac{1}{2 \theta} \mathbb{E}\left(e^{-2 \theta Y_{i}}\right) v_{*}^{2}-\left(\frac{1}{2 \theta}-\frac{\beta}{\sigma^{2}}\right){ }_{2} F_{2}\left(1,1 ; \frac{3}{2}, 2 ; \frac{\theta}{\sigma^{2}} v_{*}^{2}\right) v_{*}^{2} .
\end{aligned}
$$

By combining (175) and (180), Lemma 9 is proven.

\section{APPENDIX M \\ PROOF OF LEMMA 10}

The proof of Lemma 10 consists of the following two cases: Case 1: If $|v| \geq v_{*}$, (83) tells us that

$$
H(v)=-\gamma v^{2} .
$$

Hence, Lemma 10 holds in Case 1.

Case 2: $|v|<v_{*}$. Because $H(v)$ is an even function and $H(v)=-\gamma v^{2}$ holds at $v= \pm v_{*}$, to prove $H(v) \geq-\gamma v^{2}$ for $|v|<v_{*}$, it is sufficient to show that for all $v \in\left[0, v_{*}\right)$

$$
H^{\prime}(v)<\left[-\gamma v^{2}\right]^{\prime}=-2 \gamma v .
$$

Hence, the remaining task is to prove that (182) holds for $v \in\left[0, v_{*}\right)$.

After some manipulations, we can obtain from (87) that

$$
\left(\mathrm{mse}_{\infty}-\beta\right) G\left(\frac{\sqrt{\theta}}{\sigma} v_{*}\right)=\mathrm{mse}_{\infty} \mathbb{E}\left(e^{-2 \theta Y_{i}}\right) .
$$

Because $G(\cdot)>0$ is an increasing function, it holds for all $v \in\left[0, v_{*}\right)$ that

$$
\begin{aligned}
\left(\mathrm{mse}_{\infty}-\beta\right) G\left(\frac{\sqrt{\theta}}{\sigma} v\right) & <\left(\mathrm{mse}_{\infty}-\beta\right) G\left(\frac{\sqrt{\theta}}{\sigma} v_{*}\right) \\
& =\operatorname{mse}_{\infty} \mathbb{E}\left(e^{-2 \theta Y_{i}}\right) .
\end{aligned}
$$

One can obtain (182) from (84) and (184). Hence, Lemma 10 also holds in Case 2. This completes the proof.

\section{APPENDIX N}

\section{PROOF OF LEMMA 11}

We need the following lemma in the proof of Lemma 11

Lemma 12. $\left(1-2 x^{2}\right) G(x) \leq 1$ for all $x \geq 0$.
Proof. Because $G(0)=1$, it suffices to show that for all $x>0$

$$
\left[\left(1-2 x^{2}\right) G(x)\right]^{\prime} \leq 0 .
$$

We have

$$
\begin{aligned}
& {\left[\left(1-2 x^{2}\right) G(x)\right]^{\prime} } \\
= & -\frac{1}{x^{2}} e^{x^{2}} \int_{0}^{x} e^{-t^{2}} d t+\frac{1}{x}-4 x^{2} e^{x^{2}} \int_{0}^{x} e^{-t^{2}} d t-2 x .
\end{aligned}
$$

Because $e^{-t^{2}}$ is decreasing on $t \in[0, \infty)$, for all $x>0$

$$
\int_{0}^{x} e^{-t^{2}} d t \geq \int_{0}^{x} e^{-x^{2}} d t=x e^{-x^{2}}
$$

Hence,

$$
-\frac{1}{x^{2}} e^{x^{2}} \int_{0}^{x} e^{-t^{2}} d t+\frac{1}{x} \leq 0 .
$$

Substituting (188) into (186), (185) follows. This completes the proof.

Now we are ready to prove Lemma 11

Proof of Lemma 11] The function $H(v)$ is continuously differentiable on $\mathbb{R}$. In addition, $H^{\prime \prime}(v)$ is continuous everywhere but at $v= \pm v_{*}$. Since the Lebesgue measure of those time $t$ for which $V_{t}= \pm v_{*}$ is zero, the values $H^{\prime \prime}\left( \pm v_{*}\right)$ can be chosen in the sequel arbitrarily. By using Itô's formula [69, Theorem 7.13], we obtain that almost surely

$$
\begin{aligned}
& H\left(V_{t}\right)-H(v) \\
= & \int_{0}^{t} \frac{\sigma^{2}}{2}\left[H^{\prime \prime}\left(V_{r}\right)-\theta V_{r} H^{\prime}\left(V_{r}\right)-\left(V_{r}^{2}-\beta\right)\right] d r \\
& +\int_{0}^{t} \sigma H^{\prime}\left(V_{r}\right) d W_{r} .
\end{aligned}
$$

For all $t \geq 0$ and all $v \in \mathbb{R}$, we can show that

$$
\mathbb{E}_{v}\left\{\int_{0}^{t}\left[\sigma H^{\prime}\left(V_{r}\right)\right]^{2} d r\right\}<\infty .
$$

This and [69, Theorem 7.11] imply that $\int_{0}^{t} \sigma H^{\prime}\left(V_{r}\right) d W_{r}$ is a martingale and

$$
\mathbb{E}_{v}\left[\int_{0}^{t} \sigma H^{\prime}\left(V_{r}\right) d W_{r}\right]=0, \forall t \geq 0 .
$$

Hence,

$$
\begin{aligned}
& \mathbb{E}_{v}\left[H\left(V_{t}\right)-H(v)\right] \\
= & \mathbb{E}_{v}\left[\int_{0}^{t} \frac{\sigma^{2}}{2}\left[H^{\prime \prime}\left(V_{r}\right)-\theta V_{r} H^{\prime}\left(V_{r}\right)-\left(V_{r}^{2}-\beta\right)\right] d r\right] .
\end{aligned}
$$

Next, we show that for all $v \in \mathbb{R}$

$$
\frac{\sigma^{2}}{2} H^{\prime \prime}(v)-\theta v H^{\prime}(v)-\left(v^{2}-\beta\right) \leq 0 .
$$

Let us consider the following two cases:

Case 1: If $|v|<v_{*}$, then (76) implies

$$
\frac{\sigma^{2}}{2} H^{\prime \prime}(v)-\theta v H^{\prime}(v)-\left(v^{2}-\beta\right)=0 .
$$


Case 2: $|v|>v_{*}$. In this case, $H(v)=-\gamma v^{2}$. Hence,

$$
\begin{aligned}
& \frac{\sigma^{2}}{2} H^{\prime \prime}(v)-\theta v H^{\prime}(v) \\
= & \frac{\sigma^{2}}{2}(-2 \gamma)-\theta v(-2 \gamma v) \\
= & -\sigma^{2} \gamma+2 \theta \gamma v^{2} \\
= & -\operatorname{mse}_{Y_{i}}+\mathbb{E}\left[1-e^{-2 \theta Y_{i}}\right] v^{2} .
\end{aligned}
$$

Substituting (194) into (192), yields

$$
\mathbb{E}\left[e^{-2 \theta Y_{i}}\right] v^{2} \geq \beta-\operatorname{mse}_{Y_{i}} .
$$

To prove (195), since $|v|>v_{*}$, it suffices to show that

$$
\mathbb{E}\left[e^{-2 \theta Y_{i}}\right] v_{*}^{2} \geq \beta-\operatorname{mse}_{Y_{i}},
$$

which is equivalent to

$$
\left(\mathrm{mse}_{\infty}-\mathrm{mse}_{Y_{i}}\right) \frac{v_{*}^{2}}{\mathrm{mse}_{\infty}} \geq\left(\mathrm{mse}_{\infty}-\mathrm{mse}_{Y_{i}}\right)-\left(\mathrm{mse}_{\infty}-\beta\right) .
$$

We now prove 197). By Lemma 12, we get

$$
\left(1-\frac{v_{*}^{2} 2 \theta}{\sigma^{2}}\right) G\left(\frac{\sqrt{\theta}}{\sigma} v_{*}\right) \leq 1 .
$$

Hence,

$$
\left(1-\frac{v_{*}^{2}}{\mathrm{mse}_{\infty}}\right) G\left(\frac{\sqrt{\theta}}{\sigma} v_{*}\right) \leq 1 .
$$

By substituting (87) into (200), we obtain

$$
\begin{aligned}
& \left(\mathrm{mse}_{\infty}-\mathrm{mse}_{Y_{i}}\right)\left(1-\frac{v_{*}^{2}}{\mathrm{mse}_{\infty}}\right) G\left(\frac{\sqrt{\theta}}{\sigma} v_{*}\right) \\
\leq & \left(\mathrm{mse}_{\infty}-\beta\right) G\left(\frac{\sqrt{\theta}}{\sigma} v_{*}\right) .
\end{aligned}
$$

Because $G(x)>0$ for all $x>0$,

$$
\left(\mathrm{mse}_{\infty}-\mathrm{mse}_{Y_{i}}\right)\left(1-\frac{v_{*}^{2}}{\mathrm{mse}_{\infty}}\right) \leq \mathrm{mse}_{\infty}-\beta,
$$

which implies (197). Hence, (192) holds in both cases. Thus, $\mathbb{E}_{v}\left[H\left(V_{t}\right)-H(v)\right] \leq 0$ holds for all $t \geq 0$ and $v \in \mathbb{R}$. This completes the proof.

\section{APPENDIX O \\ PROOF OF THEOREM 6}

According to [60, Prop. 6.2.5], if we can find $\pi^{\star}=\left(Z_{1}\right.$, $\left.Z_{2}, \ldots\right)$ and $\lambda^{\star}$ that satisfy the following conditions:

$$
\begin{aligned}
& \pi^{\star} \in \Pi_{1}, \lim _{n \rightarrow \infty} \frac{1}{n} \sum_{i=0}^{n-1} \mathbb{E}\left[Y_{i}+Z_{i}\right]-\frac{1}{f_{\max }} \geq 0, \\
& \lambda^{\star} \geq 0, \\
& L\left(\pi^{\star} ; \lambda^{\star}\right)=\inf _{\pi \in \Pi_{1}} L\left(\pi ; \lambda^{\star}\right), \\
& \lambda^{\star}\left\{\lim _{n \rightarrow \infty} \frac{1}{n} \sum_{i=0}^{n-1} \mathbb{E}\left[Y_{i}+Z_{i}\right]-\frac{1}{f_{\max }}\right\}=0,
\end{aligned}
$$

then $\pi^{\star}$ is an optimal solution to (64) and $\lambda^{\star}$ is a geometric multiplier [60] for (64). Further, if we can find such $\pi^{\star}$ and $\lambda^{\star}$, then the duality gap between (64) and (68) must be zero, because otherwise there is no geometric multiplier [60, Prop. 6.2.3(b)]. The remaining task is to find $\pi^{\star}$ and $\lambda^{\star}$ that satisfy (202)-205).

According to Theorem 5 and Corollary 1 a solution $\pi^{\star}=\left(Z_{0}(\beta), Z_{1}(\beta), \ldots\right)$ to (204) is given by (89), where $\beta=$ mse $_{\text {opt }}+\lambda^{\star}$. In addition, because the $Y_{i}$ 's are i.i.d., the $Z_{i}(\beta)$ 's in policy $\pi^{\star}$ are i.i.d. Using (202), (203), and (205), the value of $\lambda^{\star}$ can be obtained by considering two cases: If $\lambda^{\star}>0$, because the $Z_{i}(\beta)$ 's are i.i.d., we have from (205) that

$$
\lim _{n \rightarrow \infty} \frac{1}{n} \sum_{i=0}^{n-1} \mathbb{E}\left[Y_{i}+Z_{i}(\beta)\right]=\mathbb{E}\left[Y_{i}+Z_{i}(\beta)\right]=\frac{1}{f_{\max }} .
$$

If $\lambda^{\star}=0$, then (202) implies

$$
\lim _{n \rightarrow \infty} \frac{1}{n} \sum_{i=0}^{n-1} \mathbb{E}\left[Y_{i}+Z_{i}(\beta)\right]=\mathbb{E}\left[Y_{i}+Z_{i}(\beta)\right] \geq \frac{1}{f_{\max }} .
$$

Next, we compute mse $_{\text {opt }}$ and $\beta=$ mse $_{\text {opt }}+\lambda^{\star}$. To compute mse $_{\text {opt }}$, we substitute policy $\pi^{\star}$ into (63), which yields

$$
\begin{aligned}
\text { mse }_{\text {opt }} & =\lim _{n \rightarrow \infty} \frac{\sum_{i=0}^{n-1} \mathbb{E}\left[\int_{Y_{i}}^{Y_{i}+Z_{i}(\beta)+Y_{i+1}} O_{s}^{2} d s\right]}{\sum_{i=0}^{n-1} \mathbb{E}\left[Y_{i}+Z_{i}(\beta)\right]} \\
& =\frac{\mathbb{E}\left[\int_{Y_{i}}^{Y_{i}+Z_{i}(\beta)+Y_{i+1}} O_{s}^{2} d s\right]}{\mathbb{E}\left[Y_{i}+Z_{i}(\beta)\right]},
\end{aligned}
$$

where in the last equation we have used that the $Z_{i}(\beta)$ 's are i.i.d. Hence, the value of $\beta=\mathrm{mse}_{\mathrm{opt}}+\lambda^{\star}$ can be obtained by considering the following two cases:

Case 1: If $\lambda^{\star}=0$, then (207) and (208) imply that

$$
\begin{aligned}
& \mathbb{E}\left[Y_{i}+Z_{i}(\beta)\right] \geq \frac{1}{f_{\max }}, \\
& \beta=\mathrm{mse}_{\mathrm{opt}}=\frac{\mathbb{E}\left[\int_{Y_{i}}^{Y_{i}+Z_{i}(\beta)+Y_{i+1}} O_{s}^{2} d s\right]}{\mathbb{E}\left[Y_{i}+Z_{i}(\beta)\right]} .
\end{aligned}
$$

Notice that (210) can rewritten as (36), which is a fixedpoint equation on $\beta$. According to Lemma 2 one root of (36) is in the set $\left(\mathrm{mse}_{Y_{i}}, \mathrm{mse}_{\infty}\right)$, which is also the unique root of (91); we denote this root as $\beta_{1}$. We choose $\pi^{\star}=$ $\left(Z_{0}\left(\beta_{1}\right), Z_{1}\left(\beta_{1}\right) \ldots\right)$, where $Z_{i}(\cdot)$ is given by (90). In addition, $\lambda^{\star}$ must be 0 in Case 1. Because $\lambda^{\star}=\beta_{1}-$ mse $_{\text {opt }}$, we get $\mathrm{mse}_{\mathrm{opt}}=\beta_{1}$, which is required in (210). Case 1 occurs if the root $\beta_{1}$ of (210) satisfies (209). We note that $\beta=\mathrm{mse}_{\infty}$ is another root of (210), but we do not pick policy $\pi^{\star}$ based on this root.

Case 2: If $\lambda^{\star}>0$, then (206) and (208) imply that

$$
\begin{aligned}
& \mathbb{E}\left[Y_{i}+Z_{i}(\beta)\right]=\frac{1}{f_{\max }}, \\
& \beta>\text { mse }_{\text {opt }}=\frac{\mathbb{E}\left[\int_{Y_{i}}^{Y_{i}+Z_{i}(\beta)+Y_{i+1}} O_{s}^{2} d s\right]}{\mathbb{E}\left[Y_{i}+Z_{i}(\beta)\right]} .
\end{aligned}
$$

When the root $\beta_{1}$ of (210) does not satisfy (209), Lemma 3 tells us that (211) has a unique root in the set $\left[\mathrm{mse}_{Y_{i}}, \mathrm{mse}_{\infty}\right)$, which is denoted by $\beta_{2}$. We choose $\pi^{\star}=\left(Z_{0}\left(\beta_{2}\right), Z_{1}\left(\beta_{2}\right) \ldots\right)$, where $Z_{i}(\cdot)$ is given by (90). Further, we choose $\lambda^{\star}=\beta_{2}-$ 
mse $_{\text {opt }}$.

Theorem 5, together with the fact that $\beta_{1}, \beta_{2} \in\left[\mathrm{mse}_{Y_{i}}, \mathrm{mse}_{\infty}\right)$ and the arguments above, implies that the selected $\pi^{\star}$ and $\lambda^{\star}$ satisfy (202)-(205). By [60, Prop. 6.2.3(b)], the duality gap between (64) and (68) is zero. A solution to (64) and (68) is $\pi^{\star}$. This completes the proof. 